\title{
Facilitating peripheral interaction: design and evaluation of peripheral interaction for a gesture-based lighting control with multimodal feedback
}

\author{
Marigo Heijboer $^{1} \cdot$ Elise van den Hoven ${ }^{2,3} \cdot$ Bert Bongers $^{2} \cdot$ Saskia Bakker $^{3}$
}

Received: 8 December 2014 / Accepted: 18 November 2015/Published online: 14 December 2015

(C) The Author(s) 2015. This article is published with open access at Springerlink.com

\begin{abstract}
Most interactions with today's interfaces require a person's full and focused attention. To alleviate the potential clutter of focal information, we investigated how interactions could be designed to take place in the background or periphery of attention. This paper explores whether gestural, multimodal interaction styles of an interactive light system allow for this. A study compared the performance of interactions with the light system in two conditions: the central condition in which participants interacted only with the light system, and the peripheral condition in which they interacted with the system while performing a high-attentional task simultaneously. Our study furthermore compared different feedback styles (visual, auditory, haptic, and a combination). Results indicated that especially for the combination feedback style, the interaction could take place without participants' full visual attention, and performance did not significantly decrease in the peripheral condition. This seems to indicate that these interactions at least partly took place in their periphery of attention and that the multimodal feedback style aided this process.
\end{abstract}

Marigo Heijboer

marigoheijboer@gmail.com

1 Department of Industrial Engineering and Innovation Sciences, Eindhoven University of Technology, 5612 AZ Eindhoven, The Netherlands

2 Department of Design, Architecture and Building, University of Technology, Sydney, P.O. Box 123, Broadway, Sydney, NSW 2007, Australia

3 Faculty of Industrial Design, Eindhoven University of Technology, P.O. Box 513, 5600 MB Eindhoven, The Netherlands
Keywords Attention - Interaction design - Mental resources · Multimodal interaction - Peripheral interaction . User evaluation

\section{Introduction}

In our everyday lives, we frequently interact with the digital world. While the human body allows for a broad range of interaction modalities to be used simultaneously, e.g., via movement of the body or sound [50], most digital interfaces rely heavily on the visual modality [10]. Interactions with a visual interface furthermore usually require a persons' full and focused attention [19, 21]. Such interactions are therefore often difficult to be performed during other activities.

In contrast, in the physical world, humans are much more adept in combining multiple tasks, especially when these tasks involve physical actions. For example, we can easily walk while making a phone call or drink from a cup while watching TV. Additionally, we can be aware of all kinds of information, such as the weather or the time of day, without consciously focusing our attention on the information sources. Activities such as walking, drinking, and processing information can be performed outside the focus of attention and instead be carried out in the background or periphery of attention. These types of activities can therefore be considered peripheral actions. Only when it is necessary, such activities may shift to the center of attention, for example when almost walking into something.

Inspired by such everyday peripheral activities, Weiser [47] has envisioned a world in which interactions with computers could similarly reside in the periphery, outside of focused attention. Weiser and Brown [48] refer to this as calm technology, technology that "engages both the center 
and the periphery of our attention, and in fact moves back and forth between the two" [48, p. 79].

In a world where technology is becoming omnipresent, designing interactions with computing technology that can reside in the periphery of attention is highly relevant. Such calm technology designs could facilitate computing systems to better fit into people's everyday life routines. Most related work has focused on conveying perceptual information in the periphery $[17,26,48]$. However, we are specifically interested in the people's ability to physically interact with digital information in their periphery, since this would allow for people to interact with systems in the periphery of their attention while being engaged in another, more important, task. These types of interactions are referred to as (physical) peripheral interactions $[3,16]$ and are intended to be performed in parallel with the user's primary task without requiring their full attention [20]. Being able to perform some physical interactions in the periphery of attention is expected to allow people to more effortlessly interact with the multiplicity of devices in their environment.

In the study presented in this paper, we evaluated a light system designed by Bongers and van den Akker [11], called the light pointer. Their system offers a novel way of interacting with light; lamps inside a room can be selected via a laser inside a pointing tool, and the lamp's brightness can subsequently be adjusted by physically moving the pointing tool. Because this system allows for interactions to take place in the physical domain as well as a multitude of feedback types during the interaction, it has the potential of guiding interactions in the periphery of attention. In this paper, we explore how the light pointer could support peripheral interaction. In this exploration, we first iteratively redesigned the light pointer interaction styles based on an exploratory experiment to select those most beneficial in supporting our aim for physical peripheral interaction. Secondly, we conducted a more extensive experiment in which we evaluated the extent to which people can interact with the redesigned light pointer interaction styles in their periphery of attention. The results of this study indicated that a combination of the redesigned interaction styles allowed for the interaction with the light pointer to take place without large performance decrements and without participants' full visual attention. This offers some indications that the interaction could at least partly take place in their periphery of attention.

\section{Literature review and related work}

In this section, divided attention theory is described, which underlies the concept of peripheral interaction; related interaction styles are addressed; and existing examples of peripheral interaction design are discussed.

\subsection{Divided attention theory}

The concept of peripheral interaction is grounded in divided attention theory, describing how we can perform different tasks simultaneously [49]. Divided attention theory describes attention as a limited amount of mental resources available to be divided over potential activities and thereby to perform these activities [28]. Everyday tasks, such as drinking from a cup, often require only few mental resources because people are experienced in performing these tasks; they have become automated processes demanding less mental resources. Therefore, multiple such tasks can be performed at the same time. However, when tasks require more mental resources, such as typing a document on the computer, fewer resources are available to allocate to other tasks. When tasks are not yet automated, Wickens' multiple resource model shows that successfully multitasking the load on mental resources and attention is dependent on the type of resources a task demands for different information channels; the required processing stage (perception, cognition, and action), the way the information is coded (the modes of representation), and the perceptual (or sensory) input and output modalities required [50, p. 329]. When different information sources compete for the same resource, the information channels might get overloaded, and the information can no longer be processed correctly. Due to this, some tasks are more suitable to be performed simultaneously compared to others [49]. For example, two visual activities can hardly be done at the same time while an auditory and a visual activity can easily be performed simultaneously.

In line with divided attention theory, we refer to the center of attention as the one activity to which most mental resources are currently allocated. The periphery of attention consists of the activities to which fewer mental resources are allocated [2]. A task is thus performed in the periphery when another task is being performed simultaneously, which requires more resources. These types of activities are defined as peripheral activities or peripheral tasks. Moreover, designing for peripheral interaction should entail interaction styles that allow for a division of attention between the designed tasks and one or more other tasks. In order to achieve this, the interaction should require only few mental resources. In their research, Mankoff et al. [32] set up and evaluated heuristics discussing comparable requirements, specifically for ambient displays that reside in the periphery of attention. These heuristics include, e.g., being unobtrusive until the display requires a person's full attention and requiring a minimal cognitive load. However, most of these heuristics are specified for informational displays instead of physical interaction styles. Although some research has focused more specifically on the design of peripheral interactions 
[3, 4, 16, 19, 25], no clear design guidelines have yet been proposed for physical peripheral interaction styles. For this reason, the next section discusses several interaction styles that could be beneficial for (physical) peripheral interaction.

\subsection{Related interaction styles}

One research area that investigates interaction styles that do not require focused attention is termed eyes-free interaction [14, 37, 51]. Similar to peripheral interaction, eyesfree interaction should not require conscious attention, leaving room for the performing of additional tasks. In their research, Cockburn et al. [14] propose that both proprioceptive target acquisition, the awareness of one's own body position in space (e.g., the orientation of the arm), and non-visual interaction commands such as physical gestures, defined as movements of the body used to convey information [46], are beneficial for eyes-free interaction. Both suggestions are related to research into gestural interaction styles. Communicative gestures, described by Pavlovic et al. [38], are used for the purpose of communicating, e.g., one's goal and can be used to communicate to a system that we want to select it. Pointing interaction styles as described in [14] could resemble the communicating of "I want that one there" [44, 45] and have been shown by to lead to quick and accurate selection of targets in a 2D plane in the absence of visual guidance [14]. Being able to be aware of one's bodily position compared to the position of a system and using interaction styles that compliment this thus seems to benefit eyes-free interaction. Manipulative gestures are used to manipulate physical artefacts in the environment [38] and are more useful when we want to interact with various functionalities of a system. For example, gestures resembling regularly conducted interactions with physical objects, e.g., swiping objects away, can be more easily conducted without visual attention (see, e.g., Pirhonen et al. [39] who implemented swiping interaction styles to switch to the next track of a music device). Since gestures are commonly used in interactions with objects in the physical world (for an overview, see [46]), employing these human motor abilities could potentially increase the simplicity of the interaction and reduce the required mental effort needed for the interaction.

Cockburn et al. [14] also took into account feedback provided by the system and suggested to provide non-visual feedback in output modalities. Adding auditory feedback can indeed reduce the necessity of having to view a task constantly [3] and has been shown to be comparable in accuracy to visual feedback [51]. Another important modality could be the haptic modality, as fewer stimuli present in the physical world can interfere with this type of feedback [36, 40]. In our research, we therefore focused on incorporating both auditory and haptic feedbacks in our design.

One problem with using either haptic or auditory feedback is that tasks taking place in the same (sensory) modality are more likely to interfere [49]. Auditory feedback for example will be less useful when a user is simultaneously listening to music. Combining feedback styles and designing a type of multimodal interaction style instead allows users to divide their attention over several sensory modalities. This could allow for an easier transition of the interaction into the periphery. Models and theoretical frameworks about multimodal interaction have for instance been developed in the field of human-computer interaction [7, 35], and in the field of semiotics [30]. Bongers and van der Veer [12] present a model to analyze and design multimodal interaction styles based on the dimensions of interaction layers, modes, and modalities.

Bongers and van der Veer [12] stress that designing multimodal interaction styles can greatly benefit the usability of the interactive devices. A modality can be described as a communication channel [12] and is used to distinguish between types of information that are sent or received in the interaction between humans and their environment. Modalities can be divided into sensory modalities (the human senses including tactile, kinesthetic, haptic, and proprioception) and human output modalities (incorporating, e.g., gestures).

In their model, Bongers and van der Veer [12] furthermore describe different modes for feedback and presentation of information. The manipulative mode, which is related to affordances [18], shows the action through the object itself, e.g., a physical button that affords being pressed [24]. The authors suggest that this type of interaction requires a lower cognitive load and thus fewer attentional resources.

Based on the theories of eyes-free interaction and multimodal interaction, we think that systems that allow for a manipulative way of interacting, such as (tangible) gestural interaction, potentially enable interactions to be performed with only few mental resources. Moreover, adding a combination of different non-visual feedback styles will potentially lead to the interaction requiring even less focused attention and can thus be performed in the periphery of attention. In our design, we therefore implemented various gestural interaction styles and auditory and haptic feedback in order to aid the performance of the interaction in the periphery of attention.

\subsection{Peripheral interaction designs}

Several studies have included the design and evaluation of calm technologies by displaying non-trivial information in 
the periphery of a person's attention [17, 26, 27, 34, 48]. While these examples focus on peripheral perception of information, a few devices have also been designed for (physical) peripheral interaction [6]. These devices offer the ability to perform physical interactions in the periphery of attention. An example of this is the physical token system, which allows for these tokens to be manipulated in the periphery and allow for easy visibility of task progress within a group [16]. StaTube [22] is a physical device displaying the availability of instant messaging contacts and that allows for setting one's own status through peripheral interaction. FireFlies [4] allows teachers to peripherally interact with an open-ended interactive system in their classroom. Whack Gestures [25] allows people to peripherally interact with their mobile device via quick gestures (e.g., striking).

Several studies have designed systems using gestural interaction styles that might be suitable for peripheral interaction as well. Brewster et al. [13] and Pirhonen et al. [39] designed devices which make use of swiping interaction styles linked to the outcome of the interaction (e.g., swiping forward means skipping to the next song). Pointing interaction styles have also been studied in several projects focusing on the design of remote controls, allowing for a "physical" interaction between the controller and the system. An early example is the development of a pointing token as a new interaction style for networked homes and distributed media [15]. Other examples include Pipet [33], supporting photograph sharing by allowing users to "suck up" pictures from a camera and shoot them to a screen, and GesturePen [44], a pointing device shaped like a stylus which can be used to transfer data easily between connected devices.

All together, several research areas have designed for gestural-based interactions that can or could potentially be used in the periphery of attention. The design of the interaction styles of our light pointer is based on the gestural interaction styles described above and aims to use these interaction styles to enable peripheral interaction.

\section{Interaction design of the light pointer}

As pointed out above, several studies have focused on the design and investigation of systems that aim to minimize the attentional burden placed on the user. The study presented in this paper investigates whether the type of physical interaction presented by the light pointer could be used in the periphery of attention and how gestural interaction styles and multimodal feedback styles could be designed in order to aid this process. A system for interaction with light, designed by Bongers and van den Akker [11], was taken as a starting point. The initial goal of this system, called the light pointer, was to develop a clear and effortless interaction for controlling lighting systems and to eventually work via a network of interconnected (light) devices inside the home $[9,11]$.

The interface consists of a handheld device (the light pointer, containing a laser pointer operated by a touch sensor at the bottom, an accelerometer, and a switch) and a lamp that contains a light sensor, an LED, and a small speaker, also see Fig. 1 and [11]. Interaction feedback can be provided via the LED, the speaker, and through a vibrotactile actuator inside the light pointer. People can adjust the brightness of the lamp in the system by moving the light pointer as required (e.g., tilting, rotating). All together, this system offers possibilities of integrating feedback in different modalities and allows for a gesturebased interaction.

As mentioned before, some related work aims to support physical peripheral interaction, but no clear design guidelines are available. Since gesture interfaces are often employed in these related designs [13, 14, 25, 37, 39, 44], we think that the light pointer is a suitable device to use for studying the feasibility of peripheral interactions with a gestural interface. As the light pointer was originally not developed as a peripheral interaction design however, we found it important to run an exploratory experiment with the original light pointer design and interaction styles before conducting our final experiment. The aim of this exploratory experiment was to redesign the light pointer interaction such that it could potentially be interacted with in the periphery of attention. We therefore explored which gestures and which types of feedback would be most suitable for peripheral interaction.

\subsection{Exploratory experiment}

The goal of this experiment was to evaluate what type of gestural mapping styles and feedback styles would be most suitable to enable participants to interact with the light pointer in their periphery of attention. Additionally, we were interested in participants' preferences and in the extent to which different gestural interaction styles supported interactions with the light pointer. Twelve participants (seven males and five females, mean age $=22.6$ years, $\mathrm{SD}=6.8$, range $18-43$ ) were asked to perform four predetermined tasks with the light pointer consecutively: (1) setting the lamp's light to the brightest level, (2) setting the light to the least bright level, (3) setting the light to the middle brightness level, and (4) turning the lamp off. Interactions were performed using two different gestural mapping styles based on [41] (rotating the wrist around its axis and tilting the wrist with the hand palm facing sideways). Moreover, two different feedback types were provided based on [36] (transition, a 
Fig. 1 Light pointer (left) and additions to the lamp fitting (right)
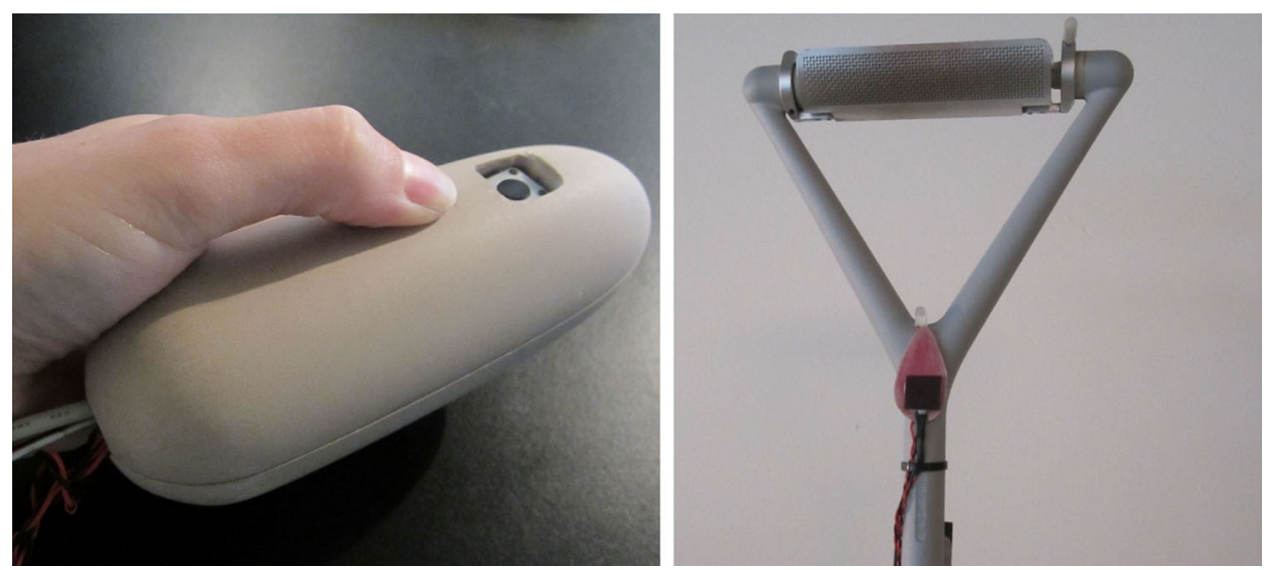
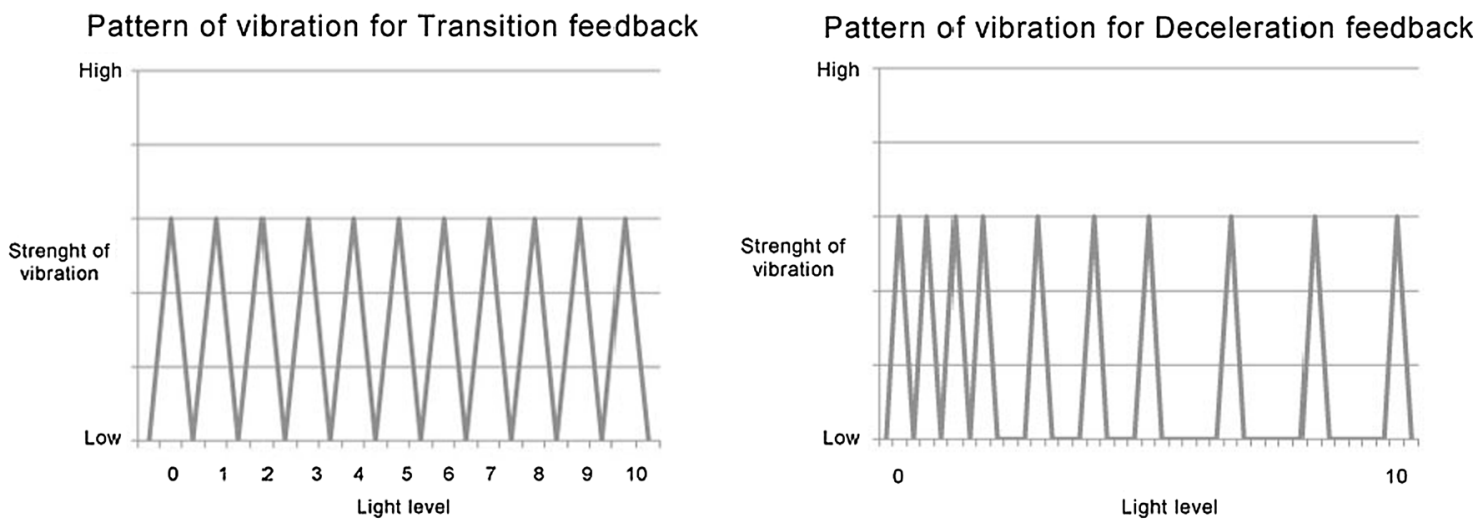

Fig. 2 Transition feedback, each peak represents one signal (left), and deceleration feedback (not per brightness level), each peak represents one signal (right)

haptic and audio signal indicating changes between brightness levels, and deceleration, offering a smaller gap between haptic and audio signals in the lowest brightness levels, see Fig. 2).

Participants performed these interactions in two different situations: they either only interacted with the light pointer, or they performed these interactions while they were reading a short story out loud. These latter interactions could potentially take place in the periphery of attention. Each participant explored the different gestural mapping styles and the different feedback styles in each of these two situations. Results of this exploratory experiment indicated that eight out of 12 participants preferred the tilting mapping style; the rotating movement was found less comfortable and less natural. Moreover, participants were able to keep performing the tasks quite well while simultaneously reading a story, showing that the interactions could potentially be performed in the periphery of attention. Lastly, most participants did not notice nor fully understand the feedback provided to them, although some who did notice it used it as a guideline to know that the system was responding. We therefore concluded that the feedback styles needed to be adapted to offer cues for the most important steps (e.g., turning the light pointer off and turning the brightness to the highest level) for the final experiment.

\subsection{Interaction with the light pointer}

Based on the results of our exploratory experiment, the gestural and multimodal interaction styles of the original light pointer were redesigned to match our goal of enabling people to interact with the light pointer in their periphery of attention. This section will explain the final light pointer interaction style.

\subsubsection{Gestural interaction}

Interactions with the light pointer consist of two stages: (1) selecting the lamp that one wants to adjust and (2) setting the brightness of this lamp, also see Fig. 3. Both interactions require a different type of gesture to be performed. A lamp can be selected by activating the laser inside the light pointer via the touch button on the bottom of the light 

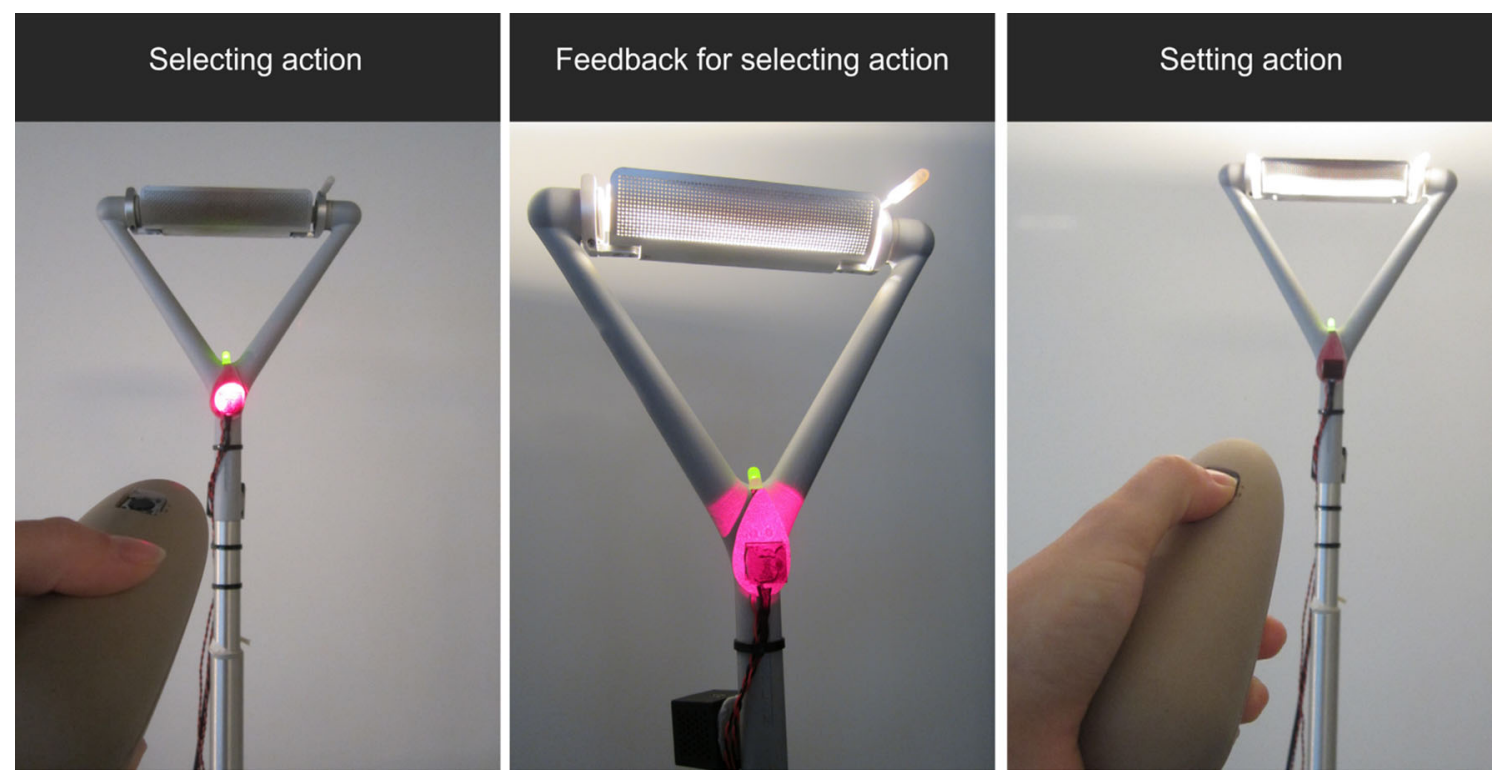

Fig. 3 Light pointer; selecting action (left), close-up view of correctly selecting the lamp, and the visual feedback of both the LED and the lamp turning on (middle) and setting action (right)

pointer and pointing it toward the light sensor mounted on the lamp. This type of gesture can be categorized as a communicative gesture; pointing to indicate that this is the preferred lamp. We refer to this as the selecting action. Once the lamp has been selected, the brightness of the lamp can be adjusted by pressing the button on top of the light pointer and simultaneously tilting the device. Moving the pointer upwards increases the lamps' brightness, while moving the pointer downwards decreases the brightness. Letting go of the button selects the current brightness. This type of gesture can be categorized as a manipulative gesture; the light of the lamp is physically manipulated in accordance with the movement of the light pointer. We refer to this as the setting action. The angle of movement is sensed by an accelerometer inside the device. Both the light pointer and the lamp are controlled by a Phidget Interfacekit ${ }^{1}$ which is connected to an Apple iMac running the Max/MSP/Jitter software. ${ }^{2}$ For a full technical explanation, see [11].

\subsubsection{Feedback styles}

The technical aspects and design of the interaction styles of the light pointer offer the possibility of feedback in different modalities: visual, auditory, and haptic feedback (see Table 1 for an overview of the technical design and accompanying interaction styles). The explanations of the various modalities in Table 1 refer to those that could be

\footnotetext{
${ }^{1}$ See www.phidgets.com, last accessed December 6, 2014.

${ }^{2}$ See www.cycling74.com, last accessed December 6, 2014.
}

adjusted during the experiment; the action of turning the laser on did not offer any pre-designed feedback, only inherent feedback. This type of feedback therefore is not mentioned as a "sensory modality." This section describes the details of the feedback styles that were used in our final experiment.

Different types of feedback can be given during the selecting and the setting action. When selecting the lamp, a visual, an auditory, or a haptic cue can be provided as confirming feedback. The visual feedback was provided by a green LED mounted on the lamp. The auditory feedback consisted of a soft clicking noise emitted from the speaker mounted on the lamp. Lastly, as haptic feedback, a strong vibration was emitted from a vibrotactile actuator inside the light pointer when the lamp was selected.

For the setting action, no specific visual feedback was designed, since we felt the light changes in the lamp itself would always be clear enough. For the auditory and haptic modality, a new type of feedback was designed based on data gathered in the exploratory experiment; participants mostly used the feedback to understand that the system was responding and to interpret whether they had turned the brightness all the way up or down. For this reason, feedback was designed to offer a short but strong vibration (haptic feedback) and a loud click (auditory feedback) when reaching the highest or lowest brightness level. More gently, texture such as vibrations (haptic feedback) and clicking sounds (auditory feedback) was offered for the brightness levels in between (see Fig. 4 for an illustration). Due to the vibration of the vibrotactile actuator, a buzzing sound was also emitted while haptic feedback was present. 
Table 1 Overview of the link between the user actions, the technical aspects of the system translating these actions into system actions, and the accompanying feedback styles (inherent and designed) and modalities; the selecting action exists of two system actions (activating laser and activating lamp) and is thus divided accordingly

\begin{tabular}{|c|c|c|c|c|c|c|}
\hline Order & Task (user actions) & Sensor (system input) & System action & $\begin{array}{l}\text { Inherent } \\
\text { feedback }\end{array}$ & $\begin{array}{l}\text { Designed } \\
\text { feedback }\end{array}$ & $\begin{array}{l}\text { Sensory } \\
\text { modality }\end{array}$ \\
\hline \multirow[t]{3}{*}{1} & \multirow[t]{3}{*}{$\begin{array}{l}\text { Selecting (touching sensor, aiming } \\
\text { pointer at light sensor, and } \\
\text { pressing button on top of pointer) }\end{array}$} & $\begin{array}{l}\text { Touch sensor } \\
\text { (activates laser) }\end{array}$ & $\begin{array}{l}\text { Turn on laser (if } \\
\text { sensor is } \\
\text { pressed) }\end{array}$ & $\begin{array}{l}\text { Laser beam } \\
\text { (shows light } \\
\text { pointer is } \\
\text { "on") } \\
\text { Relay clicking } \\
\text { sound (shows } \\
\text { activation } \\
\text { laser) }\end{array}$ & & \\
\hline & & \multirow[t]{2}{*}{$\begin{array}{l}\text { Light sensor (light- } \\
\text { dependent resistor } \\
\text { (LDR) detects laser) } \\
\text { Button (selects lamp) }\end{array}$} & \multirow[t]{2}{*}{$\begin{array}{l}\text { Activate lamp (if } \\
\text { laser is } \\
\text { detected) }\end{array}$} & \multirow[t]{2}{*}{$\begin{array}{l}\text { Laser beam } \\
\text { (shows light } \\
\text { pointer is } \\
\text { "on") }\end{array}$} & $\begin{array}{l}\text { LED (lamp is } \\
\text { selected) } \\
\text { Mouse-click } \\
\text { sound (lamp } \\
\text { is selected) }\end{array}$ & $\begin{array}{l}\text { Visual (green } \\
\text { LED) } \\
\text { Auditory } \\
\text { (click from } \\
\text { speaker) }\end{array}$ \\
\hline & & & & & $\begin{array}{l}\text { Buzzing } \\
\text { vibration } \\
\text { (lamp is } \\
\text { selected) }\end{array}$ & $\begin{array}{l}\text { Haptic } \\
\text { (vibration in } \\
\text { pointer) }\end{array}$ \\
\hline \multirow[t]{3}{*}{2} & \multirow[t]{3}{*}{$\begin{array}{l}\text { Setting (tilting pointer and releasing } \\
\text { button on top of pointer) }\end{array}$} & $\begin{array}{l}\text { Button (selects lamp) } \\
\text { Accelerometer } \\
\text { (detects tilt angle) }\end{array}$ & \multirow{3}{*}{$\begin{array}{l}\text { Set light } \\
\text { illumination } \\
\text { (via tilting light } \\
\text { pointer) }\end{array}$} & & $\begin{array}{l}\text { Brightness of } \\
\text { lamp (pointer } \\
\text { interacting) }\end{array}$ & $\begin{array}{l}\text { Visual (light } \\
\text { emitted by } \\
\text { the lamp) }\end{array}$ \\
\hline & & & & & $\begin{array}{l}\text { Audio light } \\
\text { levels } \\
\text { (pointer } \\
\text { interacting) }\end{array}$ & $\begin{array}{l}\text { Auditory } \\
\text { (audio } \\
\text { pattern from } \\
\text { speaker) }\end{array}$ \\
\hline & & & & & $\begin{array}{l}\text { Haptic light } \\
\text { levels } \\
\text { (pointer is } \\
\text { interacting) }\end{array}$ & $\begin{array}{l}\text { Haptic } \\
\text { (vibration } \\
\text { pattern from } \\
\text { pointer) }\end{array}$ \\
\hline
\end{tabular}

Next to these types of feedback, which were designed specifically by us, other forms of feedback were also present due to several aspects of the system. These included the light feedback from the lamp itself, the light of the laser, and a clicking noise emitted by the relay when activating the laser. In the following experiment, these non-designed feedback forms were held constant during all condition.

\section{Setup final experiment}

The study presented in this paper explores whether the redesigned interaction styles of the light pointer can be used in a person's periphery of attention and how physical gestural interaction and multimodal feedback support this. This section presents the setup of an experiment in which participants interacted with the light pointer, aimed at answering these research questions. These questions were explored by having participants interact with the light pointer both centrally and (potentially) peripherally, simultaneously with another, high-attentional, task and investigating which interaction styles were most beneficial for this purpose.

Based on the theories of eyes-free [14, 37] and multimodal interaction [12], we formulated a number of hypotheses regarding our participants' interactions with the light pointer. First, we hypothesized that people would be able to interact with our light pointer while simultaneously performing a different, high-attentional task (hypothesis 1a). Furthermore, we expected that the performance of this high-attentional task would be similar when performed simultaneously with interactions with the light pointer compared to when performed simultaneously with a known peripheral task (hypothesis 1b). Also, we hypothesized that participants would not need to constantly look at the light pointer during their interactions with it (hypothesis 2a).

Regarding the feedback styles, we hypothesized that performance on both the interactions with the light pointer (hypothesis 3a) and another simultaneous task (hypothesis $3 b)$ would be best when feedback from the light pointer was given in several modalities combined (visual, auditory, and haptic) and would be worst when only visual feedback 


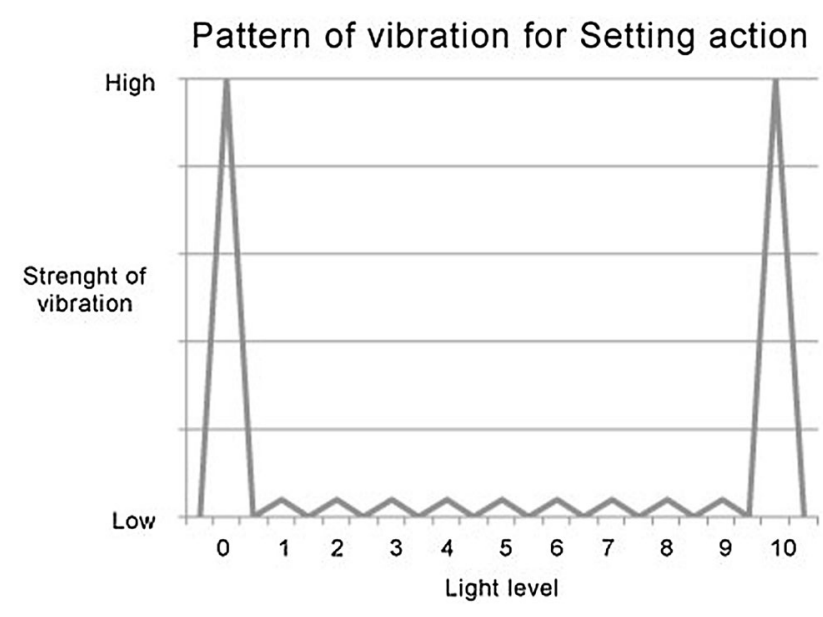

Fig. 4 Overview of feedback pattern during setting action, each peak represents a separate signal

was presented. Lastly, we hypothesized that participants needed to look at the light pointer least when combined feedback was provided, and most when only visual feedback was presented (hypothesis 4).

\subsection{Design}

To validate our hypotheses, we evaluated the participants' performance with the light pointer via different performance measurements and via assessment of video recordings made during the experiment. For the performance analysis, participants were asked to perform two tasks: the light task (involving predetermined tasks with the light pointer) and the number task (a high-attentional performance task). These tasks were either performed separately, in the central condition, or simultaneously, in the peripheral condition. The number task was also performed simultaneously with a known peripheral task (drinking from a water bottle). Next to this, performance was compared in different feedback styles: visual, auditory, haptic, and combination. While in the first three styles, feedback was only provided in one modality, and in the combination style, feedback was provided in all three modalities (a combination of the three feedback styles). For the video analysis, the amount of time participants were looking at either the light pointer interaction or the number task was assessed. This visual focus was also compared between the feedback styles. By combining these measures, we hoped to assess whether participants could interact with the light pointer in the periphery of attention.

\subsection{Participants}

Participants were students and researchers from various countries, recruited via social media, email, and word of mouth. In total, 27 people participated in the experiment. Of the total number of participants, 24 (15 males, 9 females) successfully completed the tasks and were included in the statistical analysis (mean age $=26.96$, $\mathrm{SD}=8.14$, range 19-52).

\subsection{Setting}

The experiment was conducted in a meeting room in a university building. Participants were seated at one side of a table located in the center of the room, while the experiment leader was seated on the adjacent side of the table. The lamp that was used in the experiment was placed on the opposite side of the table from where the participant was sitting (see Fig. 5), just inside the participant's visual field. A computer was located in front of the participant, which was required for the number task performed during the experiment. Video recordings were made while the participants performed the tasks.

\subsection{Measurements and tasks}

Although no clear guidelines are known for evaluating whether an interaction takes place in the periphery of attention, some indications for peripheral interaction can be derived from theory [6]. Different measurements were taken during the experiment based on these indicators to evaluate whether the participants interacted with the light pointer in the periphery of their attention. These included performance measures and visual attention measures.

\subsubsection{Performance}

Firstly, the extent to which two tasks can successfully be performed simultaneously might offer interesting insights; if two tasks can successfully be performed at once, one of these is likely performed in the periphery of attention. To measure whether participants could indeed perform the interactions with the light pointer while simultaneously performing another task, two specific tasks (the light task and the number task) were designed for this experiment. A known peripheral task (drinking from a water bottle) was also used to assess performance.

The light task involved a series of predetermined gestural interactions, combining both the selecting and the setting actions (as shown in Table 1), to be undertaken with the light pointer. Participants were asked to select the lamp (selecting action), to set the light to the maximum brightness level and subsequently to the middle brightness level (setting action). The setting action thus consisted out of two separate steps. One combination of all three steps was defined as an interaction cycle. Each interaction cycle ended by releasing the button on the light pointer, after 

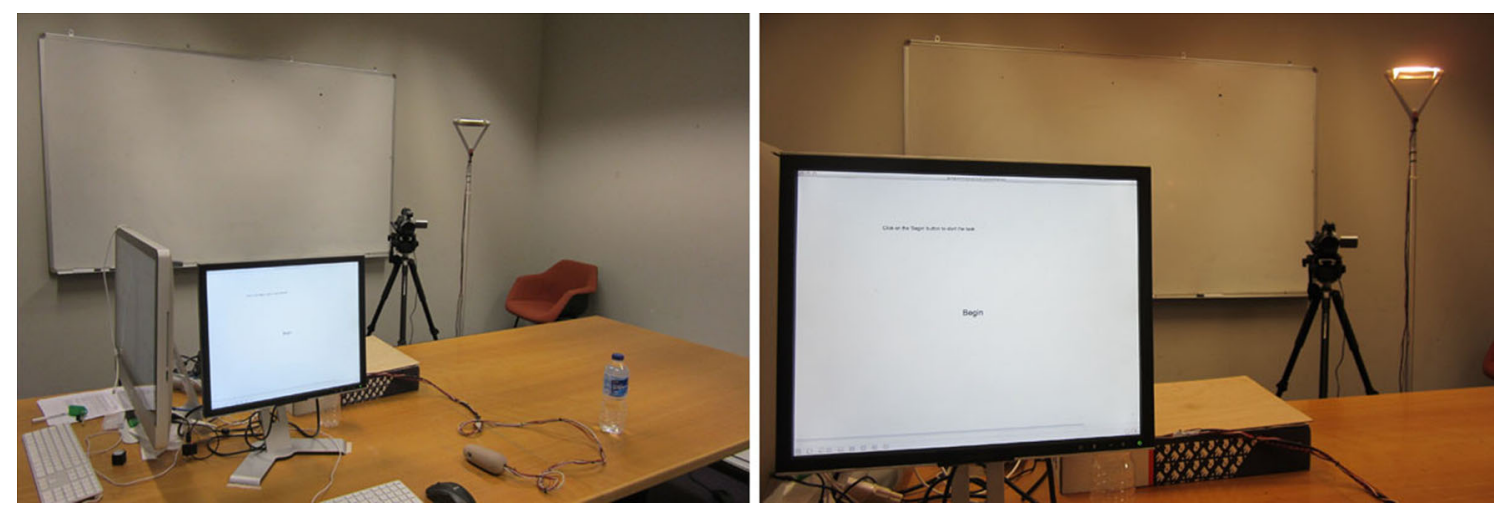

Fig. 5 Setup of the experimental room; left shows an overview of the room, and right shows the view from the participant's viewpoint

Table 2 Overview of the definitions of the various steps, actions, and interactions participants undertook when performing the light task, and how these relate to one another

\begin{tabular}{|c|c|c|c|}
\hline & Light task & & \\
\hline & Interaction cycle & & \\
\hline Type of action & Selecting action & Setting action & \\
\hline & Selecting the lamp & Setting the brightness of the lamp & \\
\hline Step in interaction & Step 1 & Step 2 & Step 3 \\
\hline & Select the lamp & Set to max brightness level & Set to middle brightness level \\
\hline Type of gestural interaction & Communicative gesture & Manipulative gesture & \\
\hline & Pointing the light pointer & Tilting the light pointer & \\
\hline
\end{tabular}

which the lamp had to be reselected. Participants were asked to repeat this interaction as often as possible over a period of $2 \mathrm{~min}$. See Table 2 for an overview of the various steps in the interaction cycle.

Performance on the light task was measured via the Max MSP software for each corresponding step of the interaction cycle. Some measurements were taken for each individual step, while others relate to the overall action or interaction cycle. Since we asked participants to repeat the cycle of interactions with the light system as often as possible, we also counted each separate interaction cycle (starting from the moment participants turned on the laser and ending when they released the top button on the light pointer). See Table 3 for an overview and explanation of all the measures.

Participants also performed a high-attentional task, called the number task. This task is an adaptation of the Go/NoGo task by Baayen and Milin [1]. The task consisted out of two stimuli: a " 1 " or a " 0 ," which were randomly generated and displayed on a computer screen. Participants were asked to react to the stimulus " 1 " by pressing on the space bar, but refrain from pressing the space bar when the stimulus " 0 " was displayed. Each task consisted out of 100 trials in the peripheral condition (lasting $2 \mathrm{~min}$ ) and of 50 trials in the central condition (lasting $1 \mathrm{~min}$ ). During each trial, one of the stimuli was displayed on the computer screen for $250 \mathrm{~ms}$. After the digit disappeared, participants had $900 \mathrm{~ms}$ left to respond before the new digit appeared. These display times were derived from studies using the sustained attention response task (SART) [29], which is similar to this number task.

For this experiment, we specifically decided upon a secondary task that has a high-attentional resource demand (as also used in [31]); it cannot be performed well without focusing attention toward it. A further advantage of the number task is that it has clear and objectively measurable performance indicators. Comparable tasks (Go/NoGo tasks and SART tasks) are often used for depletion of attentional resources measurements in experiments that try to seek for ways of regenerating one's attentional resources afterward [8], indicating that it is an inherently highly attentional task. The reading task that was used earlier is a more familiar task for participants and might in itself not require high cognitive attention. It would therefore be harder to draw conclusions regarding the cognitive attention available for other tasks. Moreover, the measurements for the reading task were more subjective and therefore harder to interpret correctly at all times. See Table 3 for an explanation of the performance measurements of the number task. 
Table 3 Overview of performance measures of the light task

\begin{tabular}{|c|c|}
\hline Performance measure & Explanation \\
\hline \multicolumn{2}{|l|}{ Light task } \\
\hline Amount of interaction cycles & The amount of times participants performed one interaction cycle during the task period \\
\hline Select duration & $\begin{array}{l}\text { The time it took a participant between pointing at the target area and starting the setting } \\
\text { process in milliseconds }\end{array}$ \\
\hline Setting duration & The time it took a participant to complete the setting action \\
\hline Percentage of correct select & The percentage of interactions in which a participant correctly selected the lamp \\
\hline $\begin{array}{l}\text { Percentage of maximum } \\
\text { brightness level }\end{array}$ & $\begin{array}{l}\text { The percentage of interactions in which a participant correctly reached the maximum } \\
\text { brightness level }\end{array}$ \\
\hline $\begin{array}{l}\text { Percentage of middle brightness } \\
\text { level }\end{array}$ & $\begin{array}{l}\text { The percentage of interactions in which a participant correctly reached the middle } \\
\text { brightness level }\end{array}$ \\
\hline \multicolumn{2}{|l|}{ Number task } \\
\hline Reaction time & Response time to the digits in milliseconds starting from the onset of the stimulus \\
\hline Percentage of mistakes & Percentage of the digits participants reacted to incorrectly \\
\hline
\end{tabular}

To be able to compare performance on the light task to tasks taking place in the periphery of attention, participants were also asked to perform another task while simultaneously performing the number task, a known peripheral task. Bodily actions such as drinking from a mug are easily performed in the periphery of attention and thus require less visual and cognitive attention than the primary task [3]. Therefore, during this task, participants were asked to pick up a water bottle next to them, bring the bottle to their mouth and take a sip, put the bottle back, and let go of the bottle. Participants were asked to repeat this action while performing the number task (but without performing the light task). No performance measurements were derived from the known peripheral task itself.

\subsubsection{Visual attention}

We defined the center of attention as the activity that requires the most mental resources. Based on this, a potential indicator for peripheral interaction is the amount of visual attention allocated to two different tasks. The task that requires the most visual attention is likely carried out in the center of attention, while a simultaneously performed second task is expected to be carried out in the periphery. To measure this, video recordings were made during the experiment, in order to analyze the participants' visual attention while performing both tasks. Two categories of codes were used in our video analysis coding scheme: direction of visual focus and duration of visual focus (see Table 4).

\subsection{Procedure}

The experiment consisted of the following three phases: introduction, interaction, and debriefing, also see Fig. 6. All together, the experiment took about $35 \mathrm{~min}$.
Table 4 Overview of coding scheme for visual attention

\begin{tabular}{ll}
\hline Coding category & Behavioral response \\
\hline Direction of visual focus & Lamp \\
(Where do participants focus & Light pointer \\
their attention toward for the & Computer \\
largest part of the action?) & Other \\
& Unclear \\
Duration of visual focus (How & Constantly \\
much of the interaction time & Most of the time \\
is the participant looking at & Half of the time \\
the interaction?) & Most of the time not \\
& Never \\
\hline
\end{tabular}

In the introduction phase of the experiment, participants were asked to read and sign a consent form. They were then explained about the light and number tasks (I) and were given the opportunity to shortly practice both tasks (P). In these practice rounds, participants had the opportunity to ask questions for clarification.

In the interaction phase of the experiment, the number task (NT), light task (LT), and known peripheral task (PT) were performed in the earlier described central and peripheral conditions. Each condition consisted of a number of task session that lasted about $2 \mathrm{~min}$. In the central condition, these sessions involved only the light task or only the number task, which are thus expected to be performed in the center of attention. In the peripheral condition, participants performed two tasks simultaneously in each session: the first four sessions combined the light task and the number task, and the fifth session combined the known peripheral task and the number task. Since the number task could not be performed without focused attention, the other tasks would likely be performed in the periphery of attention. All participants started with two task 


\begin{tabular}{|c|c|c|c|c|c|c|c|c|c|c|}
\hline \multicolumn{2}{|c|}{ INTRODUCTION } & \multicolumn{2}{|c|}{ CENTRAL BEGIN } & \multicolumn{5}{|c|}{ PERIPHERAL } & CENTRALEND & DEBRIEFING \\
\hline 1 & $P$ & NT & LT & $N T+L T$ & $N T+L T$ & $N T+L T$ & $N T+L T$ & $\mathrm{NT}+\mathrm{PT}$ & LT & $Q$ \\
\hline
\end{tabular}

Fig. 6 Overview of experiment divided into two central and one peripheral conditions ( $I$ introduction, $P$ practice round, $M$ mood, $N T$ number task, $L T$ light task, $P T$ known peripheral task, $Q$ questionnaire); feedback varied between different light tasks

sessions in the central condition: one with the light task and one with the number task (central begin condition). Subsequently, each participant performed five task sessions in the peripheral condition. At the end of the experiment, each participant conducted one task session of the light task in the central condition (central end condition). Participants performed the light task in the central condition twice, in order for us to investigate whether performance had changed due to a learning effect. Since the number task was not expected to show a large learning effect, this task was not performed at the end of the experiment. See Fig. 6 for an overview of these task sessions.

As explained before, four different styles of feedback can be provided by the light pointer (visual, auditory, haptic, and combination). In the peripheral condition, the participants were subjected to each feedback style consecutively. The order of the feedback styles was counterbalanced between participants. Participants were explained that they would receive feedback from the light pointer, but not that this feedback would vary between tasks. In the central condition, only the combination feedback style was presented. Based on research stating that (a combination of various forms of) non-visual feedback can reduce the need for visual attention [12, 14, 49], we hypothesize the combination feedback style would lead to the most accurate performance in the central condition. Differences found in the experiment, if any, could therefore not be attributed to a low performance in the central condition to begin with.

In the debriefing phase of the experiment, participants were asked to fill in some demographic information including age, gender, and experience level (indicating how much experience a person had with gestural-based games) and were given the opportunity to discuss the light pointer and the nature of the experiment with the experiment leader.

\section{Results}

The aim of the final experiment described in this paper was to evaluate whether gestural interactions with the light pointer can be performed in the periphery of attention. Furthermore, we aimed to explore which feedback styles are most suitable for peripheral interaction. In this section, the data gathered in our experiment with the light pointer will be reported. For each measure (light task, number task, and visual attention), results concerning peripheral interaction with the light pointer and results concerning the different feedback styles will be discussed separately. The first category shows results comparing the central condition of each measure to the different peripheral conditions in order to explore whether performance differed between both conditions. The second category compares the four feedback styles to explore whether the different feedback styles influenced performance.

\subsection{Light task}

The first measure that may indicate peripheral interaction with the light pointer is the participants' performance on the light task in the central and peripheral condition. This task involved a series of predetermined actions participants were asked to perform (see Sect. 4.4.1 for more detail). We expected that this performance would not differ between the two conditions, indicating that people could effectively interact with the light pointer while they were performing another task simultaneously.

In order to assess whether the interactions in the light task were performed comparably in the peripheral and the central conditions and whether there were differences between the feedback styles (visual, auditory, haptic, and combination), repeated measures ANOVAs were conducted on the various assessors of performance per experimental condition separately. These assessors were parts of the interactions undertaken and include amount of interaction cycles, select duration, setting duration, percentage of correct select, percentage of maximum brightness level, and percentage of middle brightness level (see Table 3). The experimental conditions regarding each feedback style were counterbalanced to prevent any influence due to a learning effect of the tasks.

For these analyses, mean values were calculated per participant and per condition. From these mean values, interactions during which laser errors occurred were omitted. Covariates' age, gender, and experience level were not significant and therefore not included in the final analyses.

\subsubsection{Learning effect light task}

To assess whether results found in this experiment might be due to a learning effect within the light task, participants 
were asked to perform the light task in the central condition, without performing the high-attentional task, at the beginning (central begin) and at the end of the experiment (central end). Analysis of various performance measures indeed indicated that participants were better able to interact with the light task after practice during the experiment. Results showed they were both faster and able to perform more interaction cycles. However, since this the different feedback modalities were counterbalanced, this learning effect should not have influenced our results.

\subsubsection{Peripheral interaction with the light pointer}

The various indicators of performance on the light task will be discussed separately in this section, and performance in the central and the peripheral condition will be compared to indicate specifically which interactions could be performed comparably in both conditions. For these measurements, we compared the peripheral conditions to the central end condition rather than to the central begin condition. In the central end condition, participants were best trained, and finding an effect would be most difficult. Performance measures of all participants were included in the analyses unless stated otherwise for a specific measurement. Moreover, while the covariates age, experience level, and gender were taken into account for each test, they will only be discussed and presented in the analysis if a significant effect was found.

Experience level showed a significant effect in the amount of interaction cycles' analysis $[\mathrm{F}(4.76)=10.68$, $p<0.001$ ), and was included in the analysis to control for the effect of prior experience. Analysis showed that the effect of amount of interaction cycles was significant $[F(4.76)=10.68, p<0.001]$. Participants were able to finish more complete interaction cycles in the central condition (Mcentral $=38.00, \mathrm{SE}=2.34$ ) compared to all the peripheral conditions (Mvisual $=30.00$, SEvisual $=2.44 ; \quad$ Mauditory $=30.25, \quad$ SEauditory $=2.26$; Mhaptic $=30.13, \quad$ SEhaptic $=2.44 ; \quad$ Mcombination $=27.25$, SEcombination $=2.21$, all $\mathrm{p}<0.02$ ). Participants were thus faster at performing multiple gestures with the light pointer when attention was focused solely on the light pointer.

In the analysis of percentage correct select, three participants scored very differently (significantly lower) than the others, namely the participants in a higher age group than the other participants. Only for this measurement, these participants were therefore excluded. Also, gender significantly influenced the data $[\mathrm{F}(1.18)=9.19$, $p=0.007]$ and was included in the analysis to control for this effect of gender. With these adjustments, percentage of correct select showed a significant effect $[\mathrm{F}(2.71$, $48.80)=5.99, p=0.002]$. Pairwise comparisons showed

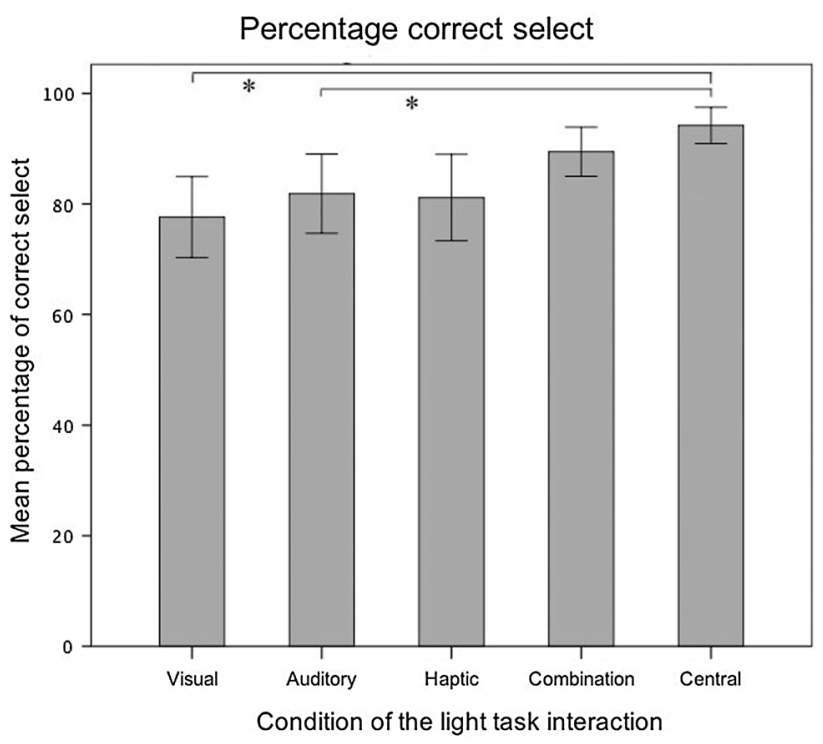

Fig. 7 Percentage of times the lamp was correctly selected in milliseconds for the different feedback styles and the central condition (two conditions linked by the "**" differ significantly from each other); error bars represent $95 \%$ confidence intervals

that participants correctly selected the light significantly more often in the central condition (Mcentral $=93.60$, SEcentral $=1.66$ ) than in the peripheral conditions in which participants used the visual (Mvisual $=78.82$, SEvisual $=2.47, p<0.001)$ and the auditory feedback style (Mauditory $=80.76$, SEauditory $=3.41, p=0.021$ ). The difference between the central condition and the peripheral condition with both the haptic feedback style (Mhaptic $=79.64$, SEhaptic $=3.89, p=0.105)$ and the combination feedback style (Mcombination $=88.79$, SEcombination $=2.23, p=0.315$ ) was not significant, see Fig. 7. This indicates that in these peripheral conditions, participants' performance approached the performance in the central condition.

The effect of select duration was also significant $[\mathrm{F}(2.5$, $41.7)=10.09, p<0.001]$. Pairwise comparisons showed that participants performed this part of the selecting action significantly faster in the central condition (Mcentral $=692.3$, SEcentral $=26.18)$ compared to the peripheral conditions with visual feedback (Mvisual $=1093.59$, SEvisual $=83.63, \quad p=0.002)$ and haptic feedback (Mhaptic $=853.7$, SEhaptic $=37.60, p=0.012)$. The difference between the speed of selection in the central condition and the peripheral condition with auditory feedback was not exactly, but close to, significant (Mauditory $=941.0, \quad$ SEauditory $=60.69, \quad p=0.051) . \quad$ The difference between the central condition and the combination feedback style (Mcombination $=787.4$, SEcombination $=25.81)$ was not significant $(p=0.074)$ see Fig. 8 . This indicates that in this peripheral condition, 


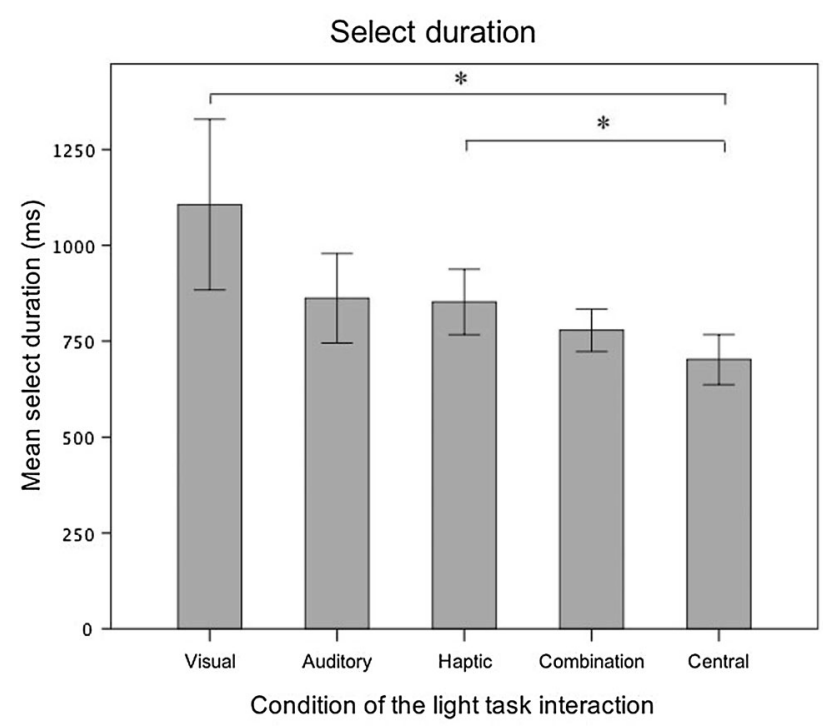

Fig. 8 Mean select duration in milliseconds for the different feedback styles and the central condition (two conditions linked by the "*" differ significantly from each other); error bars represent $95 \%$ confidence intervals

participants' performance approached the speed of selecting in the central condition.

When reviewing the data for the setting action, it became clear that there was one participant who could not accurately reach the maximum brightness level, even in the central conditions, due to wrist problems. This participant was therefore excluded from the analyses of the percentage of maximum brightness level. The final analysis showed a significant overall effect of percentage of maximum brightness level $[\mathrm{F}(4.84)=7.8, \quad p<0.001]$. Pairwise comparisons showed that in the central condition (Mcentral $=96.31$, SEcentral $=1.07$ ), participants reached the maximum brightness level significantly more often than in the peripheral conditions with visual feedback (Mvisual $=66.34, \quad$ SEvisual $=7.15, \quad p=0.002), \quad$ auditory feedback $\quad$ (Mauditory $=75.01, \quad$ SEauditory $=7.22$, $p=0.039$ ), and haptic feedback (Mhaptic $=72.91$, SEhaptic $=6.15, p=0.005$ ). However, the difference between the central condition and the peripheral condition with the combination feedback style (Mcombination $=78.95$, SEcombination $=6.67$ ) was not significant ( $p=0.088)$; see Fig. 9. Efficiency of performance of participants on this part of the action in the combination feedback style thus approached performance in the central condition.

The last two assessors, percentage of middle brightness level and setting duration, showed an overall significant effect in the analysis $\{\mathrm{F}(4.88)=3.27, p=0.015]$ and $[\mathrm{F}(2.12,27.61)=4.22, \mathrm{p}=0.022]$, respectively $\}$. However, since pairwise comparisons did not show any

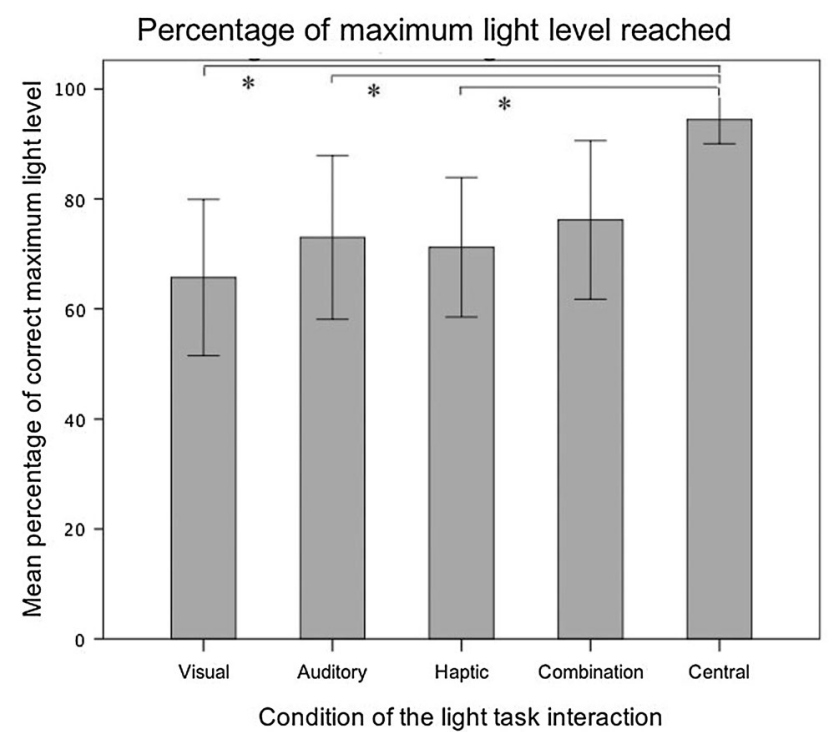

Fig. 9 Mean percentage of correct maximum brightness level in percentage for the different feedback styles and the central condition (two conditions linked by the "*" differ significantly from each other); error bars represent $95 \%$ confidence intervals

significant differences between the conditions, it is not clear where the differences actually lie without further research.

These results partly support hypothesis $1 \mathrm{a}$; in three out of the six measurements, performance on the light task in one or more peripheral conditions approached performance in the central condition, indicating participants were able to perform the light tasks well even when performing a highattentional task simultaneously. These results were mostly visible in the combination condition, supporting hypothesis $3 \mathrm{a}$ which states that performance on the interactions with the light pointer will be best when feedback is offered in different modalities simultaneously.

\subsubsection{Different feedback styles}

Performance on the light task was also compared between peripheral conditions (visual, auditory, haptic, and combination) to evaluate the suitability of different feedback styles for peripheral interaction. No significant differences between conditions were found for performance indicators amount of interaction cycles, setting duration, percentage of maximum brightness level, or percentage of middle brightness level. However, pairwise comparisons of both percentage of correct select (see Fig. 10) and select duration (see Fig. 11) showed a significant difference between the combination and the visual feedback style ( $p=0.037$ and $p=0.037$, respectively). When using the combination feedback style, participants selected the lamp correctly significantly more often (Mcombination $=88.79$, 


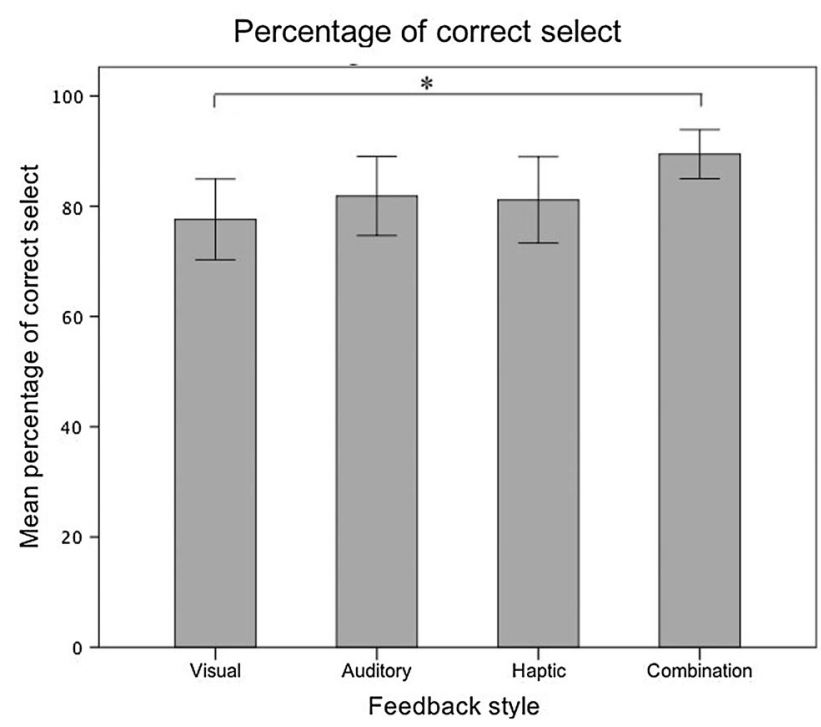

Fig. 10 Percentage of times the lamp was correctly selected per feedback style (two conditions linked by the "*” differ significantly from each other); error bars represent $95 \%$ confidence intervals

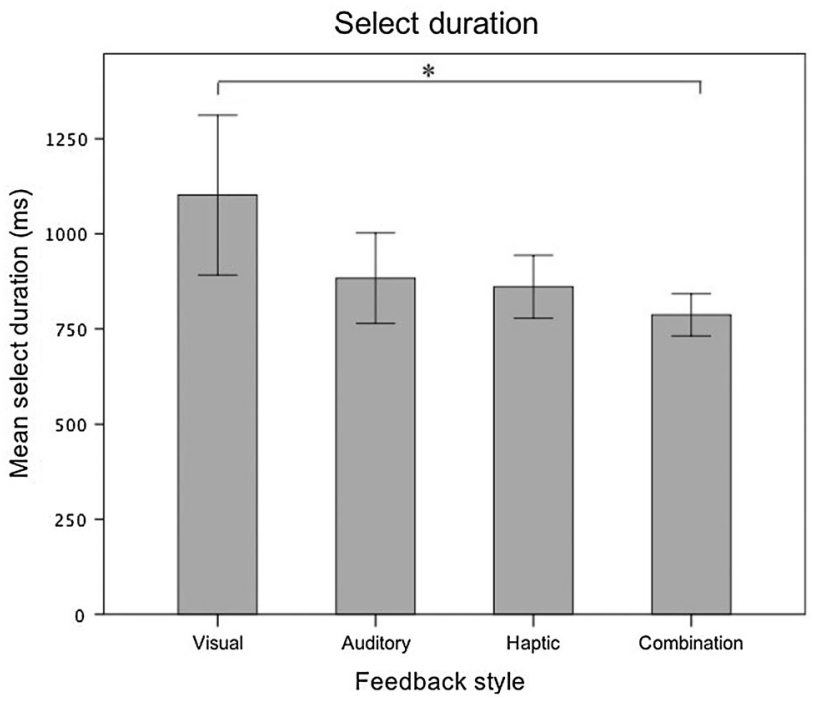

Fig. 11 Mean select duration per feedback style (two conditions linked by the "*" differ significantly from each other); error bars represent $95 \%$ confidence intervals

SEcombination $=2.23 ; \quad$ Mvisual $=78.82, \quad$ SEvisual $=2.47$ ), and they did this significantly faster (Mcombination $=787.4$, SEcombination $=25.81$; Mvisual $=1093.59$, SEvisual $=83.63$ ) than when using the visual feedback style. These results indicate that providing multimodal feedback increases performance compared to only visual feedback; however, it is unclear whether auditory and haptic feedback may ensure the same benefits. The results partly support hypothesis $3 \mathrm{a}$, which states that performance will be best when a combination of feedback in different modalities is offered.

\subsection{Number task}

A second measure for peripheral interaction with the light pointer is participant's performance on a secondary task, in this case the number task. This task consisted of a highattentional Go/NoGo task (see Sect. 4.4.1 for more detail). To assess whether the performance on the number task was affected by the simultaneous performance on the light task, a repeated measures ANOVA was conducted on the performance assessors of the number task, reaction time and percentage of mistakes separately and per experimental condition.

Initial assessment of the reaction times of participants showed some false positives (e.g., when participants pressed the spacebar too late and data were recorded for the following stimulus), namely reaction times in the peripheral conditions smaller than the fastest reaction time in the central condition $(243 \mathrm{~ms})$. These reaction times were therefore recorded as mistakes.

\subsubsection{Peripheral interaction with the light pointer}

Since we were interested in finding out whether performance on the number task was comparable in the peripheral and the central conditions, both reaction time and percentage of mistakes were compared between these conditions. For the number task, the central condition was performed before all peripheral conditions.

Analysis of participants' reaction time on the number task showed a significant effect $[\mathrm{F}(5.90)=59.49$, $p<0.001]$. Pairwise comparisons showed that when performing the light task simultaneously, participants were significantly slower in responding to the number task compared to the central condition (when only the number task was performed). Reaction time for the central condition (Mcentral $=361.9$, SEcentral $=8.3$ ) was significantly lower than reaction time for the peripheral conditions (Mvisual $=516.8$, SEvisual $=14.5 ;$ Mauditory $=548.0$, SEauditory $=12.4 ; \quad$ Mhaptic $=550.9, \quad$ SEhaptic $=14.5$; Mcombination $=554.3$, SEcombination $=13.3$; Mknown peripheral $=447.1, \quad$ SEknown $\quad$ peripheral $=11.8, \quad$ all $p<0.001)$. Reaction time in the known peripheral condition was also significantly faster than in the other peripheral conditions (all $p<0.01$ ), indicating the known peripheral task was more easily combined with the number task.

The data also showed a significant effect of percentage of mistakes $[\mathrm{F}(3.26,71.81)=23.30, p<0.001]$. Comparisons indicated that participants made significantly fewer mistakes in the central condition ( $5 \%$ of the actions; Mcentral $=5.0$, SEcentral $=1.8$; all $p<0.01)$ and in the known peripheral condition ( $3 \%$ of the actions; Mknown peripheral $=2.7, \quad$ SEknown $\quad$ peripheral $=0.5 ; \quad$ all 
$p<0.001)$ compared to the peripheral conditions when interacting with the light pointer. The difference between the known peripheral condition and the central condition was not significant. Still, participants only made mistakes in $17-21 \%$ of the actions in all light tasks, indicating performance only decreased by about $15 \%$ compared to the central condition.

Results of both performance measurements indicated that performance on the number task while performing the light task is not comparable to the same performance when drinking from the water bottle, contradicting hypothesis $1 \mathrm{~b}$. This shows that performing the light task in the periphery of attention is not as easy as performing a known peripheral task.

\subsubsection{Different feedback styles}

Performance on the number task in the different feedback styles was also compared, but none of the feedback styles differed significantly from each other. Offering multimodal feedback in this case does not seem to lead to better performance on the secondary task. This is in contradiction with hypothesis $3 \mathrm{~b}$, which states that performance on the number task will be best when feedback was combined.

\subsection{Visual attention}

In this section, we discuss the results from the behavioral coding assessment of videos recorded of participants performing the light task. By measuring visual attention next to performance, we will be able to determine better how (visual) attention is divided and how this affects performance; being able to perform an interaction without requiring visual attention can indicate that the interaction is performed in the periphery of attention.

To assess visual attention, a detailed analysis of the participants' interactions with the light pointer was conducted based on video material of the experiments. These videos were observed by the first author and analyzed for two consecutive parts of the interaction separately (selecting action and setting action), because both actions require a different type of gesture and response from the participants and are guided by different types of feedback. When reviewing the video material, the selecting action was measured from the moment the participant activated the laser until the button on top of the light pointer was pressed, and the setting action was measured from the moment the participant pressed this button until the button was released again. Each interaction part was coded according to the coding scheme shown in Table 4. Before the analysis, all data that included laser errors of the system were again omitted (e.g., when the laser was not working or the lamp could not be easily reselected after being in one of the highest brightness levels). Next, the amount of times each behavioral code was presented in each feedback style was analyzed. Because participants were almost never observed to look at the light pointer (in only three of the cases in the selecting action), this behavioral coding was combined with "looking at the lamp" and defined as "looking at the light pointer interaction."

\subsubsection{Peripheral interaction with the light pointer}

Interactions across all peripheral conditions were first evaluated together, to assess how many of the selecting and setting actions demanded participants' visual attention (i.e., when participants were looking at the light pointer during the interaction). With regard to direction of visual focus (see Table 4), analyses of the video material showed that participants mostly directed the majority of visual attention toward computer (i.e., the number task) in both the selecting (55\%) and setting (77\%) actions. This indicates that for most interactions with the light pointer, the majority of visual attention was directed away from the light pointer.

To analyze more specifically how visual attention was divided, the duration of visual focus (see Table 4) was also assessed. As evident from Fig. 12, during $46 \%$ of the selecting action and during $70 \%$ of the setting action, participants looked at the interaction with the light pointer. For an additional $9 \%$ (selecting action) and $7 \%$ (setting action), participants looked away from the interaction most of the time. These numbers show that the majority of interactions with the light pointer were performed outside of the visual focus of attention either completely or for the most part, especially during the setting action. This could

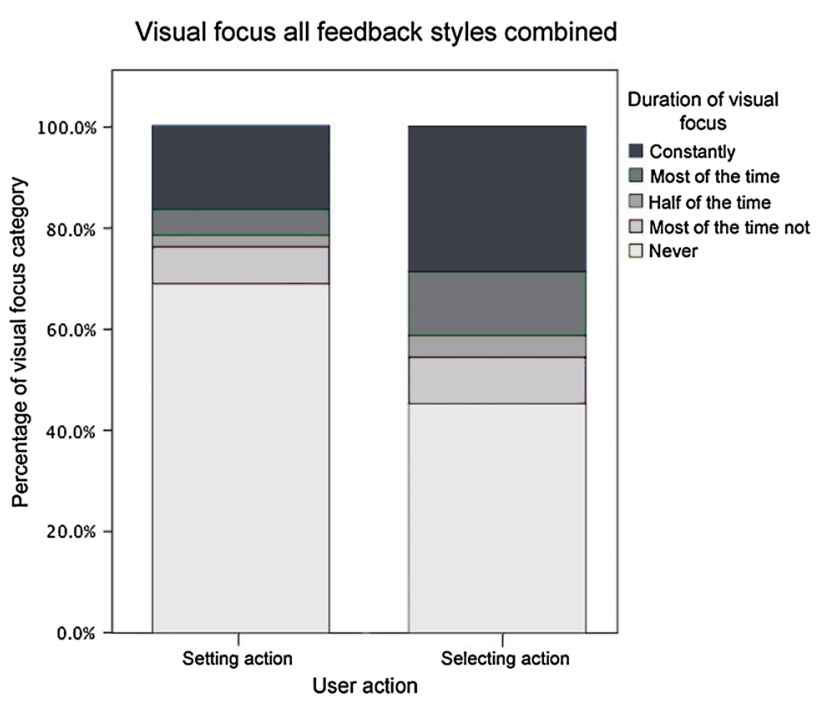

Fig. 12 Percentage of interactions being viewed for various durations of visual focus, divided in selecting and setting actions 


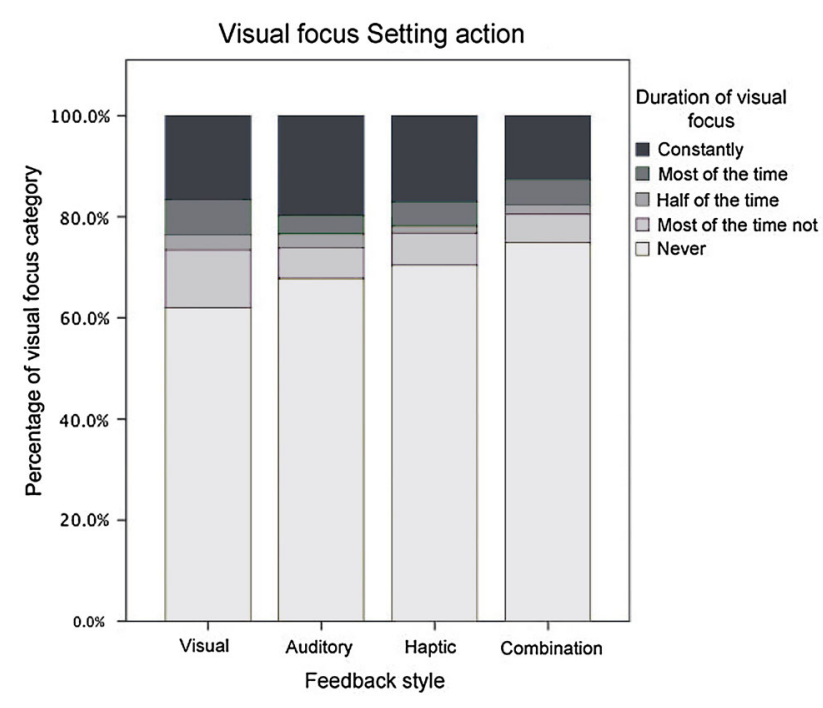

Fig. 13 Percentage of interactions being viewed for various durations of visual focus, per feedback condition, in the setting action

indicate that the participant's visual attention was in these cases focused on the number task and therefore that the light task may have been performed in the periphery of the attention. These results support hypothesis $2 \mathrm{a}$, which states that participants will not need to constantly look at the light pointer while they are interacting with it.

\subsubsection{Different feedback styles}

The observation that participants did not always need to look at the light pointer or lamp to interact with it is promising, since it may indicate that only few mental resources were required for the light task. We were furthermore interested in how the different feedback styles contributed to this. The graphs in Figs. 13 and 14 therefore show the results of the video analysis in the category "duration of visual focus," for each feedback style separately.

As evident from the graphs in Figs. 13 and 14, in all feedback styles, participants never looked at the light pointer interactions for a large amount of all interactions. Besides this, differences were also visible between the different feedback styles. For the setting action, the percentage of interactions that were not viewed by participants was highest in the combination feedback style (76\%) compared to the other feedback styles $(72 \%$ for the haptic, $68 \%$ for the auditory, and $63 \%$ for the visual feedback style). In order to discover whether the combination feedback style scored significantly different from the visual feedback style in this regard, a Chi-square test was conducted comparing visual focus of both feedback styles. Results of this test indicated that people's visual attention in the combination feedback style differed significantly from that in the visual feedback

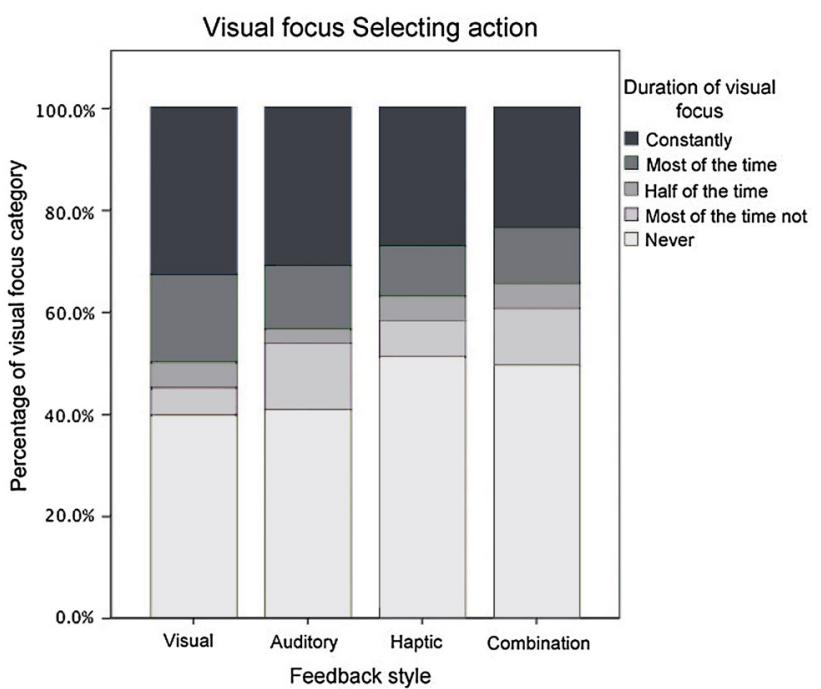

Fig. 14 Percentage of interactions being viewed for various durations of visual focus, per feedback condition, in the selecting action

style $\left[\chi^{2}(4)=25.62, p<0.001\right]$. Visual attention in the combination feedback style also differed significantly from visual attention in the auditory feedback style $\left[\chi^{2}\right.$ (4) $=14.14, p=0.006]$, but not from the haptic feedback style $\left[\chi^{2}(4)=5.46, p=0.244\right]$.

For the selecting action, participants never looked at the interaction with the light pointer for $53 \%$ of the separate interactions in the haptic feedback style, closely followed by the combination feedback style $(51 \%)$. This percentage was lower for the auditory $(41 \%)$ and the visual $(40 \%)$ feedback style. Several Chi-squared tests were conducted to investigate whether the combination feedback style differed significantly from the other feedback styles offered. Results indicated that, again, participants' visual attention in the combination feedback style differed significantly from that in the visual feedback style $\left[\chi^{2}\right.$ $(4)=35.16, p<0.001]$. Similar to the setting action, visual attention in the combination feedback style also differed significantly from the auditory feedback style $\left[\chi^{2}\right.$ (4) $=16.46, p=0.002]$, but not from the haptic feedback style $\left[\chi^{2}(4)=7.21, p=0.125\right]$.

The results of the video material thus indicate that the feedback provided by the combination feedback style was more successful than mere visual or auditory feedback in allowing participants to perform the light task outside of their visual focus, and requiring the least amount of mental resources, in both parts of the interaction (setting and selecting). However, the combined feedback was not more beneficial in this regard than the haptic feedback. In addition to this, the visual feedback presented seemed to support the peripheral interaction least during most parts of the interaction. Together, the results partly support hypothesis 4 ; in that, the combination feedback allowed 
people to focus less visual attention on the light task and that the visual feedback required most visual attention. However, the hypothesis cannot be completely accepted, since the haptic feedback style seemed to offer the same benefits as the combination feedback style. Moreover, these results show that although performance on the interactions with the light system was often highest in both the haptic and the combined feedback styles, in these conditions the least amount of visual attention was needed to perform the required interactions (correctly).

\subsection{Qualitative data}

Apart from the formal video analysis described above, we also qualitatively evaluated the video data. In this analysis, we observed the participants' behavior and recorded the remarks they made during the experiment. These observations and remarks provided insight into how the participants interacted with the light pointer and whether these interactions potentially took place in the periphery of the attention and allowed us to explore several other aspects of peripheral interaction.

\subsubsection{Peripheral interaction with the light pointer}

Although not having to focus visual attention on a task can indicate it is being performed in the periphery of attention, peripheral interaction should also allow for the interaction to shift to the center of attention when required (e.g., when something is going wrong), which is in line with the theory of calm technology [48]. This is comparable to interactions in the physical world; while people can usually drink coffee while reading a paper (action is performed in the periphery), the action is shifted to the center of attention when, e.g., coffee is spilled and attention is required to reduce further mistakes. The following example supports this aspect in the interaction with the light pointer:

One participant is performing the light task in the fourth peripheral condition, which is the visual feedback style in this case. At the same time, this participant is performing the number task, while most of the time looking at the computer. For at least seven interaction cycles in a row, the participant selects the lamp and sets its brightness level correctly without ever looking at the lamp or the light pointer. However, at some point, the participant fails to select the lamp two times in a row. When the participant notices this, attention is quickly and almost completely directed toward the lamp in order to reselect the lamp correctly. Once the participant had confirmed everything was working again, attention was shifted back to the number task.
A statement of this participant is interesting in this regard: "I lost it", indicating the rhythm of selecting the lamp was lost. This example shows that when this "rhythm" was present, the participant knew where to aim the light pointer toward without having to look at the interaction. However, when something unexpected happened (loosing this rhythm), the task with the light pointer needed to shift to the center of attention in order to correct the interaction, similar to the coffee mug example. Although attention was focused on the number task most of the time, the false selecting action could be detected in the periphery of attention, and full attention was quickly directed toward this interaction and back to the number task as soon as this was resolved.

\subsubsection{Different feedback styles}

Participants were also observed reacting to the different feedback styles in different ways, mostly depending on the order in which the feedback styles were presented. What is most interesting, although performance was on average lowest for the visual feedback style as indicated above, analysis of people's behavior does indicate that especially when presented with the first peripheral condition, participants highly relied on the visual LED feedback as well. An example is the following; in the first peripheral condition, after the central condition (haptic feedback style in this case), a participant focused visual attention towards the lamp and thought the system was not responding because the LED did not turn on (mentioned "this is so not working...”). Only after starting the setting action, and understanding the haptic feedback, the participant understood that the lamp was indeed selected. Although performance measurements described earlier indicate that presenting only the visual feedback style is least beneficial for the peripheral interaction with the light pointer, this example does indicate that the visual feedback in itself offers useful information nonetheless. Therefore, it seems that combining this feedback with other feedback styles (i.e., multimodal interaction [12]) allows for more detail in the periphery (compared to the separate feedback styles) and thus a more understandable interaction. This further supports hypothesis $3 \mathrm{a}$ which stated that performance on the light task will be best when a combination of feedback styles is offered.

\section{Discussion}

In the study presented in this paper, we explored whether an interactive light system, the light pointer, could be interacted with in the periphery of attention. Furthermore, we aimed to explore to which extent different feedback 
styles contributed to this potential for peripheral interaction. We therefore conducted an experiment in which 24 participants interacted with our light pointer, either in a central condition (in which they only interacted with the light pointer) or in a peripheral condition (in which they simultaneously interacted with the light pointer and conducted a high-attentional task). Different measurements were used to find indicators for peripheral interaction with the light pointer. Among other things, we observed whether or not participants were looking at the light pointer during the interaction, and we assessed the participant's performance in their interactions with the light pointer in the two conditions.

\subsection{Measuring peripheral interaction}

Since it is difficult to find hard evidence for the occurrence of peripheral interaction [6], we focused our analysis on a number of indicators of peripheral interaction as mentioned in the literature.

First, an indicator to determine whether an interaction could be performed in the periphery of attention is the fact that at least two tasks need to be successfully performed simultaneously. To measure this, we compared performance on the two tasks (the interactions with the light pointer and the high-attentional task) when both tasks were performed at the same time with when each task was performed separately. This way, we could verify that participants were in fact performing both tasks, instead of just ignoring one of them. Our results showed that on various measurements of the interactions with the light pointer, performance did not decrease while simultaneously performing the high-attentional task, especially when a combination of feedback styles was offered. Performance on the high-attentional task did however significantly decrease when participants simultaneously interacted with the light pointer, even in comparison with simultaneously performing the known peripheral task. Still, participants only made mistakes on the high-attentional task less than $22 \%$ of the time in all peripheral conditions. If all attention was diverted away from this task and it was somewhat neglected, we would expect the mistake rate of this task to be around chance level (50\%) as indicated in [31]. Results thus show that participants were actively performing both tasks simultaneously throughout the experiment. Despite the fact that not all tasks were performed as accurately in combination as when performed alone, one of the two tasks must thus have been performed in the periphery of attention.

One explanation for the decrease in performance on the high-attentional task could be that technical errors during the experiment (the lamp interfering with the connection between the laser and the light sensor) distracted participants. These errors could be filtered out for other measurements, but not for these data. Since only a small deviation of attention from this task would likely cause mistakes, this probably largely affected overall mean performance. Performances on the high-attentional task during the interactions with the light pointer would therefore likely have been higher and the difference with the central high-attentional task smaller if fewer errors had occurred.

Second, another indicator to determine whether one of two activities could be taking place in the periphery of attention is the amount of visual attention directed towards each interaction. When visual attention is focused toward one of two activities taking place, this could indicate that the other activity is being performed in the periphery of attention. The results of our video analysis showed that during the majority of all interactions with the light pointer, participants focused visual attention away from the light pointer (e.g., toward the screen at which the high-attentional task was displayed). The interactions with the light pointer were performed without the need for focused visual attention and were thus potentially performed in the periphery of attention. This was also the case when turning the lamp on, and no light was visible in the room that could have offered an additional cue in the participants' visual periphery. This indicates that the (gestural) interaction with the light pointer in itself likely aided the peripheral interaction.

Although participants focused visual attention away from the light pointer during the majority of their interactions, they were still able to keep performing the interactions with the light pointer and were not just neglecting it. Since the performance on the high-attentional task decreased, other forms of attention (e.g., cognitive attention) were likely divided between the two tasks. However, based on the performance rate, it is unlikely that all (cognitive) attention was diverted away from the high-attentional task. Instead, participants seemed to focus the majority of their attentional resources to the high-attentional task, while directing some resources toward the interaction with the light pointer. Despite this, interactions with the light pointer in various measurements were still performed well.

These results together thus show that (1) attention was indeed divided between the two tasks, based on performance measures and visual attention directed toward performing each task, and (2) the interaction with the light pointer required less visual attention compared to the highattentional task, while performance only decreased in some of the interactions with the light pointer. This leads us to believe that out of the two tasks performed at the same time, the light task was indeed performed in the periphery of attention most of the time (while the high-attentional task was thus performed in the center of attention most of 
the time). The implemented type of designed gestural interaction seemed to be beneficial for peripheral interaction, in line with [14]. It is important to note that the indicators of peripheral interaction that we focused on cannot provide conclusive evidence for the feasibility of peripheral interaction; they should merely be seen as indicators. Still, results of several of these measurements pointed in the same direction and together accumulate evidence, indicating that many of the interactions our participants had with the light pointer indeed took place in the periphery of attention.

\subsection{Multimodal feedback}

When comparing performance and the division of visual attention in the various feedback styles (visual, auditory, haptic, and a combination of the three), several indications of the beneficial effect of multimodal feedback were found. Firstly, observations showed that when participants were presented with a combination of feedback styles (visual, auditory, and haptic), focusing visual attention away from the light pointer was supported more than when feedback was presented in only the auditory or visual modality. Secondly, several performance measurements related to selecting the lamp were significantly higher for the combined feedback than for the visual feedback only. Even though participants looked at the interactions least when feedback was combined, performance when selecting the lamp significantly increased when feedback was combined. This offers some indications that, in agreement with the multimodality theory described in [12], multimodal feedback can be beneficial in increasing both speed and accuracy while allowing interactions to reside in the periphery more easily and thus supporting peripheral interaction. Still, the haptic feedback style seemed to also offer beneficial effects for guiding visual attention away from the secondary task, and it is worth exploring the potential of this feedback modality further.

Performance related to setting the brightness of the lamp did however not significantly increase when offering multimodal feedback. When setting the lamp, participants could likely rely on their own bodily movements in space more easily (wrist tilting), as stated in [14] to be beneficial for eyes-free interactions. Therefore, offering additional feedback might be less necessary to complete this part of the light task. In comparison, when more precision was required such as when selecting the lamp, providing multimodal feedback might be more relevant. Another possibility is that participants made use of other information during this part of the interaction, such as the light of the lamp which was clearly visible in the room, making it less necessary to rely on the designed feedback.
Last, differently from our experiment, people can encounter various distractions in real-life contexts (e.g., auditory feedback is less convenient in a crowded social event). Also, while the content of the information remains constant, people move around in real-life environments, which can result in, e.g., auditory feedback being perceived differently depending on the distance between the user and the sound source in their home. In order to truly design for peripheral interaction for everyday activities, it is important to understand the context of use, e.g., by evaluating different types of (social) situations and how these would affect the necessary feedback [5]. However, especially because of the diversity of (social) situations, applying multimodal feedback (including visual feedback) can be even more convenient.

\subsection{Methodology}

In the study presented in this paper, we measured in various ways whether interactions with the light pointer were conducted in the periphery of attention. Only limited research known to the authors has focused on finding evidence for physical peripheral interaction, and no validated measurements are known to formally evaluate peripheral interaction. By translating some general indicators for peripheral interaction into specific measurements, we set up a novel method aimed at quantitatively measuring the extent to which attention is divided. We were able to consider not only visual attention and qualitative data (as is the case in most related peripheral interaction research), e.g. $[4,6,16,22,25]$, but also performance measurements on two different tasks and combined results of both measurements in a more extensive review of the interaction. Because of this, we were not only able to indicate that the interaction was likely performed in the periphery of attention (based on the division of visual attention), but we were also able to give some indication of how well participants were able to perform these interactions. Although not all measurements led to conclusive results, these types of measurements do offer more extensive insights into how tasks are performed and whether they are indeed performed in the periphery of attention (instead of merely discarded). This contributes to the existing research about peripheral interaction and offers more possibilities of investigating this type of interaction.

In order to more accurately indicate the possibility of interactions with the light pointer taking place in the periphery of attention, we also compared the performance on the high-attentional task while performing the interactions with the light pointer with the performance on the high-attentional task while performing a known peripheral task (drinking from a water bottle). This was done because even truly peripheral interactions require some mental 
resources and could thus lead to small performance decrements on a central task. Our results however showed that drinking from a water bottle was more easily done simultaneously with a high-attentional task than interacting with the light pointer was. This might be explained by the fact that the two tasks presented to participants differed too much with regard to skill level [42, 43]. While drinking from a bottle is a familiar and highly automated task, termed a skill-based task (which was the reason it was chosen to represent a known peripheral task), the interactions with the light pointer might have been too new and required explicit attention throughout this experiment (knowledgebased task). When gesture-based interactions may become more present in future applications, the tasks with a system like the light pointer might be learned more easily (rulebased task) and eventually mastered in the same way as other, more familiar tasks, are now. It might therefore be useful in future experiments to have participants gain more experience with the new system before conducting any measurements, since this could lead to a more fair comparison with other tasks. In a study by Li et al. [31], participants were able to "practice" a central and a peripheral task for about $10 \mathrm{~h}$ to coordinate their motor responses, after which performance on a similar dual task was comparable with the same tasks performed alone.

Moreover, this peripheral task was conducted in an experimental setup. In such a laboratory environment, an everyday task such as drinking from a water bottle might not be conducted on a routine basis and therefore become more focused and less fluent. Comparing the light pointer interactions with more truly everyday tasks, perhaps by slightly adjusting the environment, or with tasks that require different amounts of mental resources (e.g., having a conversation, tying one's shoe laces) might lead to a more "fair" comparison between the light pointer interaction and another peripheral task and might offer more insights into the mental resources needed for the light pointer interaction.

In our experiment, all participants performed the interactions with the light pointer in all the feedback modalities (visual, auditory, haptic, and the three combined) leading to a comparison of 24 performance measures for each condition. When reviewing the statistical data, some outliers were removed in order to not take into account participants who performed significantly better or worse than the average. This was only the case for some measures, and in most cases, only 1-2 outliers were removed. For this study, this left us with a large enough and averagely performing group of participants to explore the general indications of peripheral interaction with our light pointer.

However, and outside the scope of this particular study, in real-life people will vary in their abilities to learn new routine activities and unlearn existing routines, and peripheral interaction will be more easily facilitated for some people than for others [5]. For example, we found some indications that three people in our study who were above the age of 40 performed some tasks differently from the rest of the group. It could be that some of the required gestural interactions might have been more difficult for people of higher age groups, e.g., because of restrictions of some muscle movements as one of the older participants suggested. In future research, it would be interesting to recruit for and compare between a larger variety of age groups in order to design for systems that can be implemented in real-world situations and be used by various unique users.

Lastly, to our knowledge, only one recent study has investigated peripheral interaction styles in a laboratory study [23]. Whereas exploring peripheral interaction in situ offers insights into the interaction in real-life situations, it offers mostly qualitative data, and it is more time-consuming compared to laboratory experiments. By setting up an experimental paradigm that enabled us to investigate several elements of peripheral interaction via direct measurements, we show that it is possible to test for peripheral interaction in a laboratory setting, and that it is easier to investigate more performance indicators in this way, thereby providing stronger evidence for the feasibility of peripheral interaction. In order to specifically measure these performance indicators, researching these with specific tasks in the laboratory first was necessary. This approach also enables testing in earlier phases of the design process, when prototypes may not be robust enough for in situ testing [23]. In the future, more systems designed for peripheral interactions could be explored in this way to get a better understanding of the attentional demands of such systems.

\section{Conclusions}

In the study described in this paper, we employed a combination of techniques to evaluate the attentional capacities needed for interacting with an interactive light system in order to find out whether this gestural interaction could take place in the periphery of attention.

Firstly, we wanted to find out whether the gestural interaction styles supported by our light pointer would allow people to interact with the light pointer in the periphery of attention. Participants therefore interacted with the light pointer while performing another, high-attentional, task. Performance on the high-attentional task, and on some measurements of the interactions with the light pointer, decreased when both tasks were performed simultaneously, compared to when they were performed separately. However, in general, participants could still 
perform both tasks quite well; some parts of the light pointer interactions did not decrease in performance when the tasks were performed simultaneously and participants did not completely stop performing one of the tasks. This indicates that both tasks could, to some extent, be performed at the same time. Moreover, throughout the experiment, participants were found to focus most of their visual attention toward the high-attentional task instead of the interaction with the light pointer. This might indicate that the majority of mental resources was directed toward the high-attentional task most of the time, offering an indication that the interaction with the light pointer indeed took place in the periphery of attention.

Secondly, we were interested in the role of different types of feedback styles, offered in different modalities, on the interaction with the light pointer. More specifically, we were interested to find out whether feedback combined over different modalities (visual, auditory, and haptic in this case) would allow for an easier transition of the interaction to the periphery of attention. Results showed that performance with the light pointer, while performing the high-attentional task simultaneously, was best on several measurements when feedback combined multiple modalities. For several of these performance measurements in the combined feedback style, there was no significant difference between only performing interactions with the light pointer and performance in combination with the high-attentional task. This indicates that offering multimodal feedback styles increases performance on speed and accuracy when performing two tasks simultaneously, offering benefits for peripheral interaction. Also, some differences were found between the different feedback styles. Again, the combination of the different feedback styles seemed to be most efficient in guiding the peripheral interaction style. When provided with this feedback, participants needed to focus less visual attention on the light pointer compared to both the auditory and visual feedback styles, and performance on the light pointer interaction for some measurements was significantly better than when only provided with visual feedback.

Overall, these results indicate that participants were able to interact with the light pointer in the periphery of attention, and that providing multimodal feedback during different steps in the interaction largely supported this peripheral interaction. Moreover, this way of evaluating peripheral interaction might offer more ways of categorizing interactions as being "peripheral." Combining different measurements (visual attention and performance) allowed for a detailed assessment of the extent to which interactions with our light pointer took place in the periphery of attention than what has previously been addressed in the related literature $[4,6,16,22,25]$ and offered more insights as to whether a task was indeed still being performed in the periphery instead of being ignored. We propose that this way of assessing of interactions leads to a more extensive review and better understanding of peripheral interactions and how they should be designed.

Acknowledgments The authors would like to thank our colleague Victor Donker for his help during the project. Furthermore, thanks are due to Femke Beute, Yvonne de Kort, and Peter Ruijten for their help with the statistical analysis. Lastly, we want to thank the University of Technology, Sydney, for providing a supportive work environment. This project was carried out with the approval of UTS Human Research Ethics Expedited Review Committee; UTS HREC Ref No. 2012-345A

Open Access This article is distributed under the terms of the Creative Commons Attribution 4.0 International License (http://crea tivecommons.org/licenses/by/4.0/), which permits unrestricted use, distribution, and reproduction in any medium, provided you give appropriate credit to the original author(s) and the source, provide a link to the Creative Commons license, and indicate if changes were made.

\section{References}

1. Baayen RH, Milin P (2010) Analyzing reaction times. Int J Psychol Res 2(3):12-28

2. Bakker S, van den Hoven E, Eggen B (2010) Design for the periphery. In: EuroHaptics 2010: proceedings of the EuroHaptics 2010 special symposium. Amsteram, The Netherlands, pp 71-80

3. Bakker S, van den Hoven E, Eggen B, Overbeeke K (2012) Exploring peripheral interaction design for primary school teachers. In: Proceedings of TEI 2012. Kingston, Ontario, Canada. February 19-22, 2012, pp 245-252

4. Bakker S, van den Hoven E, Eggen B (2013) FireFlies: physical peripheral interaction design for the everyday routine of primary school teachers. In: Proceedings of the 7th international conference on tangible, embedded, and embodied interaction. Barcelona, Spain (TEI'13), pp 57-64

5. Bakker S, van den Hoven E, Eggen B (2014a) Peripheral interaction: characteristics and considerations. Pers Ubiquitous Comput 19(1):239-254

6. Bakker S, van den Hoven E, Eggen B (2014b) Evaluating peripheral interaction design. Hum Comput Interact 30(6):473-506

7. Bernsen NO (1993) Modality theory: supporting multimodal interface design. In: Proceedings of the ERCIM workshop on multimodal human-computer interaction. Nancy, pp 13-23

8. Berto R (2005) Exposure to restorative environments helps restore attentional capacity. J Environ Psychol 25(3):249-259

9. Bongers AJ (2004) Interaction with our electronic environment: an ecological approach to physical interface design. Cahier book series. Hogeschool van Utrecht, Utrecht

10. Bongers AJ (2006) Multimodal human-computer interaction: interfacing with the ecology. Interactivation-towards an e-cology of people, our technological environment, and the arts. Academisch Proefschrift, Amsterdam, pp 99-114

11. Bongers AJ, van den Akker M (2011) Light pointer. http://inter activelight.id.tue.nl/. Accessed 6 Dec 2014

12. Bongers AJ, van der Veer GC (2007) Towards a multimodal interaction space: categorisation and applications. Pers Ubiquitous Comput 11(8):609-619

13. Brewster S, Lumsden J, Bell M, Hall M, Tasker S (2003) Multimodal 'eyes-free' interaction techniques for wearable devices. 
In: Proceedings of CHI2003. Ft. Lauderdal, Florida, USA, pp $473-480$

14. Cockburn A, Quinn P, Gutwin C, Ramos G, Looser J (2011) Air pointing: design and evaluation of spatial target acquisition with and without visual feedback. Int J Hum Comput Sci 69:401-414

15. Sluis R van de, Eggen JH, Jansen J, Kohar H (2001) User interface for an in-home environment. In: Proceedings of INTERACT'01. Tokyo 2001, pp 383-390

16. Edge D, Blackwell AF (2009) Peripheral tangible interaction by analytic design. In: Proceedings of TEI 2009, pp 69-76

17. Eggen B, van Mensvoort K (2009) Making sense of what is going on around: designing environmental awareness information displays. In: Markopoulos P, de Ruyter B, Mackay W (eds) Awareness systems: advances in theory, methodology and design. Springer, London, pp 99-124

18. Gibson JJ (1979) The ecological approach to visual perception. Houghton Mifflin, Boston

19. Hausen D (2012) Peripheral interaction: facilitating interaction with secondary tasks. In: Proceedings of TEI 2012, Kingston, Ontario, Canada, pp 387-388

20. Hausen D, Butz A (2011) Extending interaction to the periphery. In: Proceedings of workshop embodied interaction: theory and practice in HCI. In conjunction with 29th ACM International Conference on Human Factors in Computing Systems (CHI 2011). http://www.medien.ifi.lmu.de/forschung/publikationen/ detail?pub=hausen2011chi. Accessed 6 Dec 2014

21. Hausen D, Boring S, Polleti J, Butz A (2012) Exploring design and combination of ambient information and peripheral interaction. In: Proceedings of the 9th International ACM Conference on Designing Interactive Systems. DIS 2012. June 11-15, 2012. Newcastle, UK. http://www.medien.ifi.lmu.de/forschung/pub likationen/detail?pub=hausen2012dis. Accessed 6 Dec 2014

22. Hausen D, Boring S, Lueling C, Rodestock S, Butz A (2012a) StaTube: facilitating state management in instant messaging systems. In: Proceedings of TEI, 2012. Kingston, Ontario, Canada, pp 283-290

23. Hausen D, Tabard S, von Thermann A, Holzner K, Butz A (2014) Evaluating peripheral interaction. In: Proceedings of the 8th International Conference on Tangible Embedded and Embodied Interaction, pp 21-28

24. Heijboer M, van den Hoven E (2008) Keeping up appearances: interpretation of tangible artifact design. In: Proceedings of NordiCHI 2008: using bridges. Lund, Sweden, pp 162-171

25. Hudson SE, Harrison C, Harrison BL, LaMarca A (2010) Whack gestures: inexact and inattentive interaction with mobile devices. In: Proceedings of TEI 2010. Cambridge, Massachusetts, USA, pp 109-112

26. Ishii H, Wisneski C, Brave S, Dahley A, Gorbet M, Ullmer B, Yarin P (1998) ambientROOM: integrating ambient media with architecture. In: conference summary of CHI 1998. ACM Press, pp 173-174

27. Ishii H, Ren S, Frei P (2001) Pinwheels: visualizing information flow in an architectural space. In: Extended Abstract of CHI'01, pp 111-112

28. Kahneman D (1973) Attention and effort. Prentice Hall, Englewood Cliffs

29. Kelly SP, Dockree P, Reilly RB, Robertson IH (2003) EEG alpha power and coherence time courses in a sustained attention task. In: Proceedings of the 1st International IEEE EMBS Conference on Neural Engineering. Italy, pp 83-86

30. Kress G, van Leeuwen TV (2001) Multimodal discourse, the modes and media of contemporary communication. Oxford University Press, Oxford

31. Li FF, Van Rullen R, Koch C, Perona P (2002) Rapid natural scene categorization in the near absence of attention. In:
Proceedings of the National Academy of Sciences of the United States of America, pp 9596-9601

32. Mankoff J, Dey AK, Hsieh G, Kientz J, Lederer S, Ames M (2003) Heuristic evaluation of ambient displays. In: Proceedings of the SIGCHI conference on human factors in computing systems, pp 169-176

33. Meerbeek B, Bingley P, Rijnen W, van den Hoven E (2010) Pipet: a design concept supporting photo sharing. In: Proceedings of the 6th Nordic conference on human-computer interaction: extending boundaries, pp 335-342

34. Motamedi N (2007) Keep in touch: a tactile-vision intimate interface. In: Proceedings of TEI'07. Baton Rouge, LA, USA, pp 21-22

35. Nigay L, Coutaz J (1993) A design space for multimodal systems: concurrent processing and data fusion. In: Proceedings of the INTERACT'93 and CHI'93 conference on human factors in computing systems, pp 172-178

36. Oakley I, O'Modhrain S (2005) Tilt to scroll: evaluating a motion based vibrotactile mobile interface. World Haptics 2005:40-49

37. Oakley I, Park J (2007) Designing eyes-free interaction. Haptic Audio Interact Des 4813:121-132

38. Pavlovic VI, Sharma P, Huang TS (1997) Visual interpretation of hand gestures for human-computer interaction: a review. IEEE Trans Pattern Anal Mach Intell 19(7):677-695

39. Pirhonen A, Brewster S, Holguin C (2002) Gestural and audio methaphors as a means of control for mobile devices. In: Proceedings of CHI 2002. Minneapolis, Minnesota, USA, pp 291-298

40. Poupyrev I, Maruyama S, Rekimoto J (2002) Ambient touch: designing tactile interfaces for handheld devices. In: Proceedings of UIST'02. Paris, France, pp 51-60

41. Rahman M, Gustafson S, Irani P, Subramanian S (2009) Tilt techniques: investigating the dexterity of wrist-based Input. In: Proceedings of CHI 2009. Boston, Massachusetts, USA, pp 1943-1952

42. Rasmussen $\mathbf{J}$ (1986) Information processing and human-machine interaction: an approach to cognitive engineering. North-Holland, Amsterdam

43. Rasmussen J, Goodstein JP, Pejtersen AM (1994) Cognitive systems engineering. Wiley, Hoboken

44. Swindells C, Inkpen KM, Dill JC, Tory M (2002) That one there! Pointing to establish device identity. In: Proceeding of the 15th annual ACM symposium on user interface software and technology, pp 151-160

45. Valkkynen P, Niemala M, Tuomisto $T$ (2006) Evaluation touching and pointing with a mobile terminal for physical browsing. In: Proceedings of NordiCHI 2006, pp 28-37

46. van den Hoven E, Mazalek A (2011) Grasping gestures: gesturing with physical artifacts. Artif Intell Eng Des Anal Manuf 25:255-271

47. Weiser M (1991) The computer for the twenty-first century. Sci Am 265:94-104

48. Weiser M, Brown JS (1997) The coming age of calm technology. In: Denning PJ, Metcalfe RM (eds) Beyond calculation: the next fifty years of computing. Springer, New York, pp 75-86

49. Wickens CD, McCarley JS (2008) Applied attention theory. CRC Press, Boca Raton

50. Wickens CD, Hollands JG, Banbury S, Parasuraman R (2013) Engineering psychology and human performance, 4th edn. Pearson, London

51. Zhao S, Dragicevic P, Chignell M, Balakrishanan R, Baudisch P (2007) earPod: eyes-free menu selection using touch input and reactive audio feedback. In: Proceedings of the CHI 07: ACM conference on human factors in computing systems. San Jose, California, USA, pp 1395-1404 